Locus-specific induction of gene expression from heterochromatin loci during

\title{
cellular senescence
}

Kosuke Tomimatsu ${ }^{1,2,11}$, Dóra Bihary ${ }^{3,12,16}$, loana Olan ${ }^{1,16}$, Aled J. Parry ${ }^{1,4}$, Stefan Schoenfelder ${ }^{4,5}$ Adelyne S. L. Chan ${ }^{1}$, Guy St. C Slater ${ }^{1}$, Yoko Ito ${ }^{1,13}$, Peter J. RuggGunn $^{4}$, Kristina Kirschner ${ }^{1,14}$, Camino Bermejo-Rodriguez ${ }^{1,15}$, Tomomi Seko ${ }^{6,7}$, Hiroyuki Kugoh $^{6,7}$, Ken Shiraishi ${ }^{8}$, Koji Sayama ${ }^{8}$, Hiroshi Kimura ${ }^{9}$, Peter Fraser ${ }^{5,10}$, Masako Narita ${ }^{1}$, Shamith A. Samarajiwa ${ }^{3}$, Masashi Narita ${ }^{1,9}$

${ }^{1}$ Cancer Research UK Cambridge Institute, University of Cambridge, Cambridge, UK ${ }^{2}$ Shiga University of Medical Science, Shiga, Japan

${ }^{3}$ MRC Cancer Unit, Hutchison/MRC Research Centre, University of Cambridge, Cambridge, UK

${ }^{4}$ Epigenetics Programme, The Babraham Institute, Cambridge, UK

${ }^{5}$ Nuclear Dynamics Programme, The Babraham Institute, Cambridge, UK

${ }^{6}$ Department of Biomedical Science, Institute of Regenerative Medicine and Biofunction, Graduate School of Medical Science, Tottori University, Yonago, Japan

${ }^{7}$ Chromosome Engineering Research Center, Tottori University, Yonago, Japan

${ }^{8}$ Department of Dermatology, Graduate School of Medicine, Ehime University, Toon, Japan

${ }^{9}$ Tokyo Tech World Research Hub Initiative (WRHI) and Cell Biology Center, Institute of Innovative Research, Tokyo Institute of Technology, Yokohama, Japan

${ }^{10}$ Department of Biological Science, Florida State University, Tallahassee, FL, USA

${ }^{11}$ Present address: Medical Institute of Bioregulation, Kyushu University, Fukuoka, Japan

${ }^{12}$ Present address: VIB-KU Leuven Center for Cancer Biology, Leuven, Belgium

${ }^{13}$ Present address: International University of Health and Welfare, Tochigi, Japan

${ }^{14}$ Present address: Institute for Cancer Sciences, University of Glasgow, Glasgow, UK

${ }^{15}$ Present address: Department of Molecular and Clinical Cancer Medicine, University of Liverpool, Liverpool, UK

${ }^{16}$ These authors contributed equally: Dóra Bihary, loana Olan.

Correspondence: M.N. (masashi.narita@cruk.cam.ac.uk); S.A.S (ss861@mrccu.cam.ac.uk); M.N. (masako.narita@cruk.cam.ac.uk) 
Abstract

Senescence is a fate-determined state, accompanied by reorganization of heterochromatin. While lineage-appropriate genes can be temporarily repressed through facultative heterochromatin, stable silencing of lineage-inappropriate genes often involves the constitutive heterochromatic mark, histone H3K9me3. The fate of these heterochromatic genes during senescence is unclear. Here we show a small number of lineage-inappropriate genes, exemplified by the LCE2 skin genes, are derepressed during senescence from H3K9me3 regions in fibroblasts. DNA FISH experiments reveal that these gene loci, which are condensed at the nuclear periphery in proliferative cells, are decompacted during senescence. Decompaction of the locus is not sufficient for LCE2 expression, which requires p53 and C/EBP $\beta$ signalling. NLRP3, which is predominantly expressed in macrophages from an open topologically associated domain (TAD), is also derepressed in senescent fibroblasts due to the local disruption of the H3K9me3-rich TAD that contains it. NLRP3 has been implicated in the amplification of inflammatory cytokine signalling in senescence and aging, highlighting the functional relevance of gene induction from 'permissive' H3K9me3 regions in senescent cells.

54

55

56

57

58

59

60 
61 Senescence is characterized by persistent proliferative arrest triggered by diverse 62 stimuli, including excessive mitotic stress induced by persistent oncogene activation 63 as in oncogene-induced senescence (OIS) ${ }^{1}$. Senescent cells secrete various factors, 64 including cytokines, growth factors, and extracellular matrix modifying enzymes, 65 which have a profound impact on the tissue environment ${ }^{2}$. The senescence66 associated secretory phenotype (SASP) can have adverse effects in vivo, particularly 67 when it persists, and contributes to aging and age-associated disorders, including 68 cancer $^{2-4}$

70 Senescence is often accompanied by alterations to the chromatin state ${ }^{5,6}$. Active 71 chromatin marks and chromatin accessibility are dynamically altered during 72 senescence, and such alterations are well correlated with the senescence-associated 73 gene expression profile, including the $\mathrm{SASP}^{7-11}$. In contrast, heterochromatic marks, 74 particularly $\mathrm{H} 3 \mathrm{~K} 9 \mathrm{me} 3$, are largely static during OIS in some contexts ${ }^{12-14}$. However, 75 the higher-order structure of the heterochromatic regions can be drastically altered 76 during senescence. For example, senescence-associated heterochromatin foci 77 (SAHFs) are formed through the spatial reorganization of pre-existing H3K9me3- and H3K27me3-marked chromatin ${ }^{12,14}$. Senescence-associated distension of satellites (SADS), which involves the decompaction of (peri-)centric constitutive heterochromatin, is also observed without alteration of $\mathrm{H} 3 \mathrm{~K} 9 \mathrm{me} 3$ and $\mathrm{H} 3 \mathrm{~K} 27 \mathrm{me} 3$

81 levels at these regions ${ }^{9,15}$. These studies suggest that the 3D structural alterations of 82 heterochromatic regions during senescence can occur without loss or gain of 83 repressive marks.

85 Epigenetic gene regulation is critical for establishing cell types and lineages. Tissue 86 specific gene silencing is typically attributed to facultative heterochromatin, marked 87 by H3K27me3. In committed progenitor cells, it has been suggested that H3K27me3 88 is involved in the silencing of lineage-specific genes that are induced upon terminal 
differentiation ${ }^{16}$. In contrast, $\mathrm{H} 3 \mathrm{~K} 9 \mathrm{me} 3$ is mainly enriched in the non-coding parts of the genome (e.g., repetitive regions) regardless of the cell lineage, consistent with its close association with constitutive heterochromatin. However, it has become evident that H3K9me3-enriched heterochromatin also contributes to silencing of genes specifically expressed in other tissues (i.e., lineage-inappropriate genes), thereby ensuring the stability of cell identity ${ }^{16,17}$. However, how such cell type specific genes are regulated during senescence is not clear.

Here we show evidence that lineage-inappropriate genes can be expressed from H3K9me3-heterochromatic domains during senescence. This senescenceassociated 'leakage' of lineage-inappropriate genes is accompanied by the physical decompaction of a subset of the heterochromatic regions, which gain active chromatin features. This is in marked contrast to the derepression mechanism for lineage appropriate genes during terminal differentiation. We propose that the selective derepression of genes embedded in heterochromatic regions represents a distinct gene expression mechanism, which transcends the epigenetic barrier in differentiated cells.

\section{Results}

\section{Aberrant expression of lineage-inappropriate genes during senescence}

While investigating our previously published transcriptomic datasets in IMR90 human diploid fibroblasts (HDFs) induced into senescence by different stressors ${ }^{18-20}$, we noticed that multiple Late cornified envelope (LCE) genes, mainly in the LCE2 subcluster, were up-regulated in oncogenic $\mathrm{HRAS}^{\mathrm{G} 12 \mathrm{~V}}$-induced senescence (RIS) or DNA damage-induced senescent (DDIS) IMR90 cells. This was surprising, since the LCE genes (consisting mainly of three sub-clusters LCE1-3) represent a tissuespecific gene cluster. The LCE gene cluster is located in the Epidermal Differentiation Complex (EDC) ( 1.5 Mb) locus on chromosome 1q21, which contains a number of 
117 cornification genes that are induced during epidermal terminal differentiation in the

118 stratum corneum ${ }^{21,22}$. These structural proteins are then enzymatically cross-linked to

119 reinforce cornification at the very late stage of epidermal differentiation. Thus, the

120 LCE genes are lineage-inappropriate in fibroblasts. Indeed, expression of genes

121 within the EDC locus was barely detectable in IMR90 cells in any of the other

122 conditions we tested, including not only the normal proliferative condition, but also

123 quiescence, transformation (via co-expression of $\mathrm{HRAS}^{\mathrm{G} 12 \mathrm{~V}}$ and the adenoviral

124 oncoprotein $E 1 A^{18}$ ), and acute DNA damage (Extended Data Fig. 1a). The p53 tumor

125 suppressor plays a critical role in senescence ${ }^{1}$, and the upregulation of most LCE

126 genes during senescence was diminished upon introduction of a short-hairpin against

127 p53 (sh-p53) in RIS cells (Extended Data Fig. 1a), reinforcing the correlation between

128 upregulation of some LCE genes in fibroblasts and the senescence phenotype. A

129 similar induction of LCE genes was also observed in multiple publicly available

130 datasets for senescent HDFs with different senescence triggers, except for a dataset

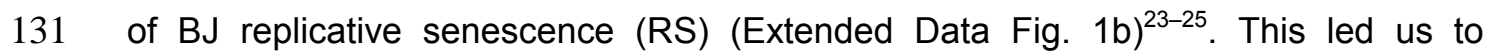

132 hypothesize that a potentially new mechanism for derepressing tissue-specific genes

133 in non-orthotopic tissues underlies the EDC gene induction during senescence.

134

135 We first validated the expression of the LCE2 proteins (encoded by LCE2A-D) in 136 IMR90 cells. While LCE2, probed by an anti-pan-LCE2 antibody ${ }^{26}$, was undetectable 137 in proliferating cells, RIS IMR90 cells exhibited a comparable amount of LCE2 to 138 differentiated human keratinocytes (Fig. 1a). Involucrin, encoded by another EDC 139 gene $I V L$, was induced during keratinocyte differentiation, as expected, but it was 140 also modestly detected in RIS fibroblasts (Fig. 1a). Consistent with the transcriptomic 141 data (Extended Data Fig. 1a), the levels of the LCE2 proteins during RIS were 142 reduced when p53 was knocked-down (Extended Data Fig. 1c). To gain insight into 143 the mechanism for the aberrant expression of these genes, we next characterized 144 the chromatin state of the EDC locus in IMR90 cells using ChIP-seq and RNA-seq 
145 datasets, which we have previously published ${ }^{12,14,20}$. We found that, in both

146 proliferative and RIS IMR90 cells, the majority of the EDC locus was enriched for 147 H3K9me3 flanked by H3K27me3 (Fig. 1b). Consistently, our RNA-seq analysis 148 revealed that the transcriptional activity of the genes within the EDC of proliferative 149 IMR90 cells was very low. Despite the apparent lack of reduction in the H3K9me3 150 coverage (if anything there was a slight increase) on the EDC during RIS (Fig. 1b, c), 151 the central region of the EDC, surrounding the LCE2 genes, became transcriptionally 152 active (Fig. 1b). We next examined the permissive nature of transcription at this 153 region by ATAC-seq (Assay for Transposase-Accessible Chromatin using 154 sequencing ${ }^{27}$ ) and H3K27ac ChIP-seq ${ }^{10}$. ATAC-seq maps chromatin accessibility, 155 whereas $\mathrm{H} 3 \mathrm{~K} 27 \mathrm{ac}$ is associated with active transcription and, together with high 156 chromatin accessibility, marks active regulatory regions (promoters/enhancers) ${ }^{28}$. 157 The EDC locus of RIS, but not proliferative, IMR90 cells exhibited H3K27ac 158 enrichment, which coincided with ATAC-seq peaks 50 kb downstream of the LCE2 159 cluster, suggesting that active enhancers are formed within the 'heterochromatic' 160 EDC during RIS, albeit modest ones (Fig. 1d). These data suggest that tissue161 specific genes can be expressed from H3K9me3-rich regions in non-orthotopic 162 tissues during senescence. In contrast, while a cluster of olfactory receptor (OR) 163 genes, another tissue-specific genes on chromosome $1 \mathrm{q} 22^{29}$, was also broadly 164 enriched for $\mathrm{H} 3 \mathrm{~K} 9 \mathrm{me} 3$, this cluster did not produce any detectable transcripts, nor 165 H3K27ac enrichment or ATAC-seq peaks in either proliferating or RIS IMR90 cells 166 (Fig. 1d). Thus, the mechanism of gene expression from H3K9me3-heterochromatin 167 appears to be locus specific.

168

169 A potential tumor suppressor activity of LCE1 (homologous to LCE2 and also 170 encoded from the EDC locus) has previously been proposed ${ }^{30}$. To test whether 171 ectopic expression of an LCE2 protein promotes replicative exhaustion in normal 172 fibroblasts, we overexpressed LCE2A in IMR90 cells and performed population 
173 doubling and colony formation assays. LCE2A-expressing cells showed a reduced

174 capacity in proliferation and colony formation (Extended Data Fig. 1d, e). These

175 results potentially suggest a functional relevance of the derepression of $L C E$ genes in

176 normal fibroblasts.

177

178 Distinct epigenetic landscapes of EDC genes in senescent cells relative to 179 keratinocytes

180 To directly compare the chromatin status of the EDC between fibroblasts and 181 keratinocytes, we performed ChIP-seq for key histone marks before and after 182 terminal differentiation in primary human keratinocytes. It has been suggested that 183 EDC genes in undifferentiated keratinocytes are facultatively silenced by the 184 polycomb repressive complexes (PRCs) and the corresponding repressive histone 185 mark H3K27me3 ${ }^{31,32}$. We confirmed these observations using ChIP-seq. In contrast 186 to IMR90 fibroblasts, H3K27me3, but not H3K9me3, was enriched across the EDC 187 locus in keratinocytes (Fig. 1b). We also confirmed that the H3K27me3 level was 188 reduced during differentiation, whereas H3K27ac-peaks were increased (Fig. 1b, c).

189 These data indicate that the nature and dynamics of the epigenetic landscape at the 190 EDC locus is different between keratinocyte differentiation (where EDC genes are 191 lineage-appropriate) and senescence in fibroblasts (where EDC genes are lineage192 inappropriate).

193

194 The EDC locus is structurally altered in senescent fibroblasts

195 Senescence is often accompanied by alterations of higher-order chromatin 196 structure ${ }^{33}$, highlighted by both heterochromatin reorganization (represented by $197 \mathrm{SAHFs}^{34}$ ) and decompaction (represented by SADS ${ }^{15}$ ) without substantial alterations 198 in H3K9me3. To characterize the chromatin structure of the EDC, we performed DNA 199 fluorescence in situ hybridization (FISH) experiments with multiple Bacterial Artificial 200 Chromosome (BAC) probes tiled across the EDC locus (Fig. 2a, bottom). In 
201 proliferating IMR90 cells, these FISH signals were highly condensed and tended to 202 be located at the nuclear periphery, which provides a repressive environment (Fig. 2b, 203 Extended Data Fig. 2a). In contrast, in RIS IMR90 cells, FISH experiments using the 204 same probes exhibited a decompacted pattern with an extended fluorescent signal 205 length (Fig. 2b, c). Although less prominent, EDC decompaction was also observed 206 in DNA damage-induced senescence (DDIS) (d7) and replicative senescence (RS) 207 IMR90 cells (Fig. 2c). It was not detected after acute DNA damage (acDD) (d1) or in 208 senescence-bypassing transformed cells expressing E1A+RAS, suggesting that the 209 EDC decompaction is specifically associated with senescence (Fig. 2c). Of note, the 210 morphology of the OR locus, which was not derepressed during RIS (Fig. 1d), 211 remained unchanged in RIS IMR90 cells (Extended Data Fig. 2b).

213 In primary keratinocytes isolated from human skin, the EDC FISH probes also 214 exhibited a compacted pattern and we failed to detect any significant decompaction 215 during differentiation (Fig. 2b, c). Interestingly, this locus was located at the nuclear 216 interior, which is often transcriptionally permissive, both before and after 217 differentiation (Extended Data Fig. 2a), consistent with a previous study showing that 218 relocation of the EDC locus from nuclear periphery to inner nuclear regions already 219 occurs during embryonic development, at least in mice ${ }^{35}$. This further supports the 220 notion that distinct mechanisms derepress the EDC genes in senescent fibroblasts 221 relative to differentiated keratinocytes.

223 We next conducted a time series analysis of the EDC morphology using IMR90 cells 224 expressing oncogenic $\mathrm{HRAS}^{\mathrm{G} 12 \mathrm{~V}}$ in a 4-hydroxytamoxifen (4OHT) inducible form 225 (ER:HRAS ${ }^{\mathrm{G} 12 \mathrm{~V}}$ ), where senescence is progressively established over $\sim 6$ days ${ }^{19}$. Our 226 FISH analysis showed that the EDC signal length became increasingly extended 227 over the time course (Fig. 2d). Similarly, LCE2 expression was also more evident at 228 later time points (Fig. 2e). Consistently, in our published microarray data ${ }^{19}$, LCE2 
upregulation was most prominent at the late stage of RIS (Extended Data Fig. 1a),

230 indicating that local decompaction of the EDC and derepressing of the LCE2 genes

231 are temporally correlated during RIS. It has been shown that SADS is established

232 during the early stages of senescence ${ }^{15}$, whereas SAHFs, which involve the

233 chromosome-wide spatial segregation of transcriptionally active (peri-SAHFs) and

234 inactive (intra-SAHFs) regions, progressively accumulate during senescence ${ }^{36}$.

235 Although decompaction of the EDC is reminiscent of SADS, the kinetics of the EDC

236 decompaction appeared to be more similar to the kinetics of SAHF formation (Fig. 2f).

237 Within RIS cell populations, the EDC was significantly decompacted in SAHF-positive

238 compared to SAHF-negative cells (Extended Data Fig. 2c). Immunolabelling analysis

239 also revealed that the majority of SAHF-positive cells were LCE2-positive (Extended

240 Data. Fig. 2d). These data reinforce a close correlation between EDC decompaction

241 and SAHF formation, although any mechanistic relationship between SADS and EDC

242 decompaction cannot be excluded. In agreement with this, in IMR90 cells, EDC

243 decompaction was most prominent in RIS IMR90 cells compared to DDIS and RS

244 IMR90 cells, where SAHF formation is modest (Fig. 2c, Extended Data Fig. 2e). Of

245 note, LCE gene induction in DDIS and RS was also weaker than in RIS (Extended

246 Data Fig. 1b) and LCE2 protein levels were undetectable or variable in DDIS or RS

247 IMR90 cells, respectively (Extended Data Fig. 2f). In addition, we have shown that

248 different HDFs develop SAHFs to varying degrees: for example, SAHF-formation was

249 weak in BJ cells, compared to IMR90 and WI38 cells during RIS (Extended Data Fig.

$2502 \mathrm{~g})^{37}$. Up-regulation of LCE2 protein levels in RIS BJ cells was marginal with no

251 significant decompaction of the EDC (Extended Data Fig. 2h, i).

252

253 We next examined a spatial correlation between SAHFs and the structure of the EDC

254 locus. In the multi-probe FISH analyses, the EDC appeared to be located at inter-

255 SAHFs 'open' regions (Fig. 2b, Extended Data Fig. 2a). To validate this, we

256 conducted FISH experiments co-staining both the EDC and OR loci (using a single 
257 probe for each locus, as depicted in Extended Data Fig. 2b, top) and found that the

258 colocalization frequency of the OR locus with SAHFs was significantly higher than

259 that of the EDC locus with SAHFs (Fig. 2g). Interestingly, both the EDC and OR loci

260 were enriched for Lamin B1 (LMNB1) and each formed a single lamina-associated

261 domain (LAD) (Fig. 2a) $)^{14}$. Lamin B1 levels are reduced during senescence ${ }^{38}$ and we

262 have previously shown that this reduction in Lamin B1 occurs predominantly from

$263 \mathrm{H} 3 \mathrm{~K} 9 \mathrm{me} 3$ regions, and that this liberation of $\mathrm{H} 3 \mathrm{~K} 9 \mathrm{me} 3$ heterochromatic regions from

264 the nuclear envelope is correlated with SAHF formation ${ }^{12}$. Indeed, the reduction in

265 Lamin B1 was particularly prominent at the OR locus, compared to the EDC locus

266 (Fig. 2a), further supporting the model that SAHF formation involves a spatial

267 reorganization of pre-existing heterochromatin ${ }^{12}$. These data suggest that at these

268 two lineage-specific gene clusters, both of which are enriched for H3K9me3 in HDFs,

269 distinct gene regulatory mechanisms operate: the EDC locus is derepressed outside

270 of SAHFs by physical decompaction, while the OR locus is incorporated into SAHFs

271 and remains condensed and silenced.

272

273 To test the direct correlation between EDC decompaction and SAHF formation, we

274 knocked-down HMGA1, which is an essential structural component of SAHFs, during

275 RIS induction (RAS+shHMGA1) (Fig. 3a) ${ }^{37}$. The EDC decompaction was partially, but

276 significantly, inhibited in RAS+shHMGA1 cells although EDC decompaction in these

277 cells was still significant compared to the basal level (Fig. 3b). RNA-seq analysis

278 revealed a trend of reversal of the derepression of most EDC genes in

279 RAS+shHMGA1 cells (Fig. 3c). Consistently, the induction of LCE2 proteins was also

280 weaker in the presence of shHMGA1 (Fig. 3d). These data collectively suggest that

281 HMGA1-dependent heterochromatin compartmentalization somehow promotes the

282 EDC decompaction/derepression possibly through an indirect mechanism. 
284 Ectopic expression of p16 (a CDK-inhibitor) is sufficient for inducing SAHF-positive 285 senescence $^{34}$. However, as reported ${ }^{39}$, these cells showed a distinct gene expression 286 profile, lacking typical persistent DNA damage response (pDDR) and inflammatory 287 senescence-associated secretory phenotype (SASP) (Fig. 3e). To further gain insight 288 into the relation between EDC decompaction and these senescence effector 289 phenotypes, we measured EDC decompaction and gene expression from this locus 290 in p16-induced senescent IMR90 cells. While less pronounced than in RIS cells, p16291 senescent cells exhibited both SAHFs and EDC decompaction (Fig. 3f, g). Our RNA292 seq data, however, showed little change in the EDC genes in p16-senescent cells, 293 compared to RIS or even DDIS, the latter in which SAHF formation and derepression 294 of the EDC genes were milder than in the RIS cells (Fig. 3h). The data suggest that 295 SAHFs and EDC decompaction are not sufficient for the EDC gene induction, which 296 probably requires additional signalling or specific transcription factor activation.

298 One candidate for such signalling is the p53 pathway: consistent with the literature ${ }^{39}$, 299 the p53 downstream target, p21, was not upregulated in p16-senescent IMR90 cells 300 (Fig. 3e). Additionally, consistent with the immunoblotting results (Extended Data Fig. 301 1c), induction of LCE2 genes (except for LCE2A) during RIS was partially p53302 dependent (Fig. 3i, j). Thus, we tested whether the LCE2 genes are direct targets of 303 p53. We reanalyzed our p53 ChIP-seq data ${ }^{18}$ and found that p53 mainly binds the 304 LCE2A gene body, concomitant with increased deposition of H3K27ac (an active 305 enhancer mark), in RIS cells (Fig. 3k). These results suggest that p53 may control at 306 least some EDC genes through directly binding to the intragenic enhancer during RIS, 307 although p53-dependent downstream signalling may also contribute. Notably, p53308 depletion had no significant impact on the EDC decompaction (Fig. 3I).

310 The level of a key transcription factor that drives the inflammatory SASP, , is 311 upregulated during $\mathrm{RIS}^{40}$. Consistent with the literature ${ }^{39}$, the SASP was largely 
312 absent in p16-induced senescent cells, where we found little upregulation of CEBPB

313 (Fig. 3e). Interestingly, induction of the LCE2 genes during RIS was inhibited by

314 depletion of C/EBP $\beta$ (Fig. 3i, j). This prompted us to map C/EBP $\beta$ binding sites in

315 RIS cells. We detected C/EBP $\beta$ peaks around the promoters of LCE2 genes,

316 suggesting that the LCE2 genes may be C/EBP $\beta$-targets. Furthermore, co-depletion

317 of p53 and C/EBP $\beta$ showed stronger effects than singular knockdown (Fig. 3j),

318 suggesting a co-regulation of LCE2 genes by these two transcription factors, at least

319 in part through direct binding. Together, our data indicate that deregulation of the

320 EDC locus consists of two steps: first, decompaction of the locus and second,

321 activation of specific signalling and/or transcription factors.

322

\section{Aberrant expression of lineage-specific genes and chromatin decompaction}

324 Chromatin accessibility has been linked with cell-type-selective regulatory elements ${ }^{41}$,

325 reflecting cell fate and maturity ${ }^{42}$. It has been shown that chromatin accessibility is 326 increased during senescence ${ }^{9,10}$. To test whether senescence-associated lineage327 inappropriate gene expression is unique to the EDC genes, we searched for similar 328 H3K9me3 domains that encompass both H3K27ac and ATAC-seq peaks in 329 proliferative and RIS IMR90 cells, using high confidence data sets of H3K9me3, 330 H3K27ac and ATAC-seq peaks (Materials and Methods).

332 While the number of $\mathrm{H} 3 \mathrm{~K} 9 \mathrm{me} 3$ peaks were relatively stable with 763 common peaks 333 (805 in proliferative cells; 874 in RIS cells ${ }^{12}$ ), we previously showed that the number 334 of ATAC-seq peaks (73,946 in proliferative cells; 238,269 in RIS cells) and H3K27ac 335 peaks $(95,464$ in proliferative cells; 117,385 in RIS cells) increase overall during RIS 336 in IMR90 cells ${ }^{10}$. Consistent with previous studies ${ }^{43}$, H3K27ac peaks mostly 337 coincided with ATAC-seq peaks (Extended Data Fig. 3a). 
339 Using the information about these active marks as well as gene expression (RNA340 seq data we previously published ${ }^{20}$ ), we characterized H3K9me3 peaks: 136 peaks 341 (defined in the RIS condition) contained both de novo ATAC- and H3K27ac peaks 342 ('permissive' H3K9me3 peaks). 110 of these peaks showed no induction of gene 343 expression, whereas 26 peaks were accompanied by significant upregulation of gene 344 expression, including the LCE genes. Thus, we grouped these H3K9me3 peaks as 345 follows: group 1 (non-permissive; 627 peaks): H3K9me3 peaks with no de novo 346 ATAC-seq and H3K27ac peaks, group 2 (110 peaks): permissive (both de novo 347 ATAC-seq and H3K27ac peaks) without gene activation, and group 3 (26 peaks): 348 permissive with gene activation (Fig. 4a, Extended Data Fig. 3b). The permissive $349 \mathrm{H} 3 \mathrm{~K} 9 \mathrm{me} 3$ peaks (group 2+3), regardless of altered gene expression, appeared to be 350 larger in width compared to the non-permissive peaks (group 1) (Fig. 4b). However, 351 group 3 of H3K9me3 peaks were significantly higher in gene density and were less 352 AT-rich than group 2 (Fig. 4c, d). Among repeat elements, group 3 peaks were 353 enriched for short-interspersed nuclear elements (SINEs), which are often found in 354 GC-rich regions and in gene promoters ${ }^{44}$ (Fig. 4e, f, Extended Data Fig. 3c). As 355 exemplified by the EDC locus mentioned above (Fig. 2a), group 3 H3K9me3 peaks 356 exhibited the least pronounced reduction of Lamin B1 during RIS (Fig. 4g-i). Thus, 357 permissive $\mathrm{H} 3 \mathrm{~K} 9$ me3 peaks with aberrant gene induction (group 3) appear to have 358 distinct features characterized by higher gene density with residual Lamin B1 359 association.

361 We next explored individual genes within group $3 \mathrm{H} 3 \mathrm{~K} 9 \mathrm{me} 3$ peaks (a list of genes 362 included in each H3K9me3-peak group can be found in Supplementary Table 1). 363 Close visual inspection revealed that some 'active regulatory regions' (i.e., co364 occurring H3K27ac and ATAC-seq peaks) overlapped permissive H3K9me3 peaks 365 either in regions with $\mathrm{H} 3 \mathrm{~K} 9 \mathrm{me} 3$ signal or within small gaps in $\mathrm{H} 3 \mathrm{~K} 9 \mathrm{me} 3$ in either the 366 proliferative or RIS conditions (Extended Data Fig. 3d). Using stringent criteria (both 
gene bodies and promoters with de novo ATAC/H3K27ac peaks entirely overlapping H3K9me3 peaks and not gaps in the H3K9me3 ChIP-seq signal), we identified 8 genes in 6 loci (PDPN, LCE2A, LCE2C, IVL, NLRP3, DCC, TMEM132B, and ZNF667) among the 38 significantly up-regulated genes during RIS in group 3

$371 \mathrm{H} 3 \mathrm{~K} 9 \mathrm{me} 3$ peaks (see highlights in Fig. 4b-i). Note, Involucrin (encoded by IVL within 372 the EDC locus) was also detected in RIS fibroblasts (Fig. 1a). To address the 373 orthotopic expression status for these aberrantly expressed genes in normal tissues, 374 we compared our RNA-seq data ${ }^{20}$ with publicly available RNA-seq data obtained 375 from The Human Protein Atlas (HPA) ${ }^{45}$. The basal expression levels of these genes 376 in IMR90 cells were mostly low and they tended to show 'tissue-enriched' expression 377 patterns, according to the HPA criteria (Fig. 5a, Extended Data Fig. 4a). Thus, the 378 H3K27ac/ATAC peaks established within H3K9me3 domains during senescence 379 may mark aberrant inductions of lineage-inappropriate genes.

381 To test whether derepression of these genes during senescence is commonly 382 accompanied by chromatin decompaction, we performed multi-probe DNA FISH 383 analysis for NLRP3, DCC and TMEM132B loci (BAC probe positions in Fig. 5b; the $384 \mathrm{H} 3 \mathrm{~K} 9 \mathrm{me}$-rich regions encompassing PDPN and ZNF667 were too small, $\sim 200 \mathrm{~kb}$ 385 and $150 \mathrm{~kb}$, respectively, for the multi-probe FISH experiment, in which each BAC 386 probe is $\sim 200 \mathrm{~kb}$ long). Similar to the EDC locus, all loci tested were highly 387 condensed in proliferative IMR90 cells, but the FISH signal lengths were significantly 388 extended in RIS cells (Fig. 5b-d, Extended Data Fig. 4b).

Decompaction of H3K9me3 domains correlates with expression of lineage-

392 To further investigate whether heterochromatin decompaction during senescence is 393 associated with lineage-inappropriate genes, we decided to take advantage of a RIS 394 model in human keratinocytes, in which the EDC is a lineage appropriate gene 
395 cluster. We first profiled EDC gene expression in RIS keratinocytes and compared to 396 the differentiation condition using RNA-seq. In contrast to RIS fibroblasts, the EDC 397 genes were widely expressed in RIS keratinocytes, exhibiting a similar pattern to 398 differentiated keratinocytes (Fig. 6a). This is consistent with previous studies showing 399 that some EDC genes are induced in senescent keratinocytes, induced by oncogenic 400 HRAS or oxidative stress ${ }^{46,47}$. Next, we examined the EDC condensation status. As a 401 comparison, we chose the DCC locus, identified in this study as differentially 402 expressed in proliferating compared to RIS fibroblasts. DCC encodes a netrin 1 403 receptor, which is expressed mainly in testis, lung and brain ${ }^{45}$. In contrast to the EDC, 404 the DCC locus was embedded in a H3K9me3 region in both IMR90 cells and 405 undifferentiated keratinocytes, but not in hES cells (Fig. 6b), suggesting that DCC 406 becomes stably heterochromatinized in both fibroblasts and keratinocytes during 407 development; thus, DCC is lineage-inappropriate in keratinocytes. We performed 408 additional FISH experiments for the DCC and EDC loci in these three cell types: hES 409 cells, fibroblasts (control and RIS conditions), and keratinocytes (control and RIS 410 conditions). Similar to IMR90 fibroblasts, we found a significant decompaction of 411 DCC in RIS keratinocytes (Fig. 6c). Consistently, the mRNA levels of DCC were 412 modestly but significantly increased in the RIS keratinocytes accompanied by the up413 regulation of key SASP factors associated with senescence (Fig. 6d). Thus, the 414 senescence-associated decompaction of lineage inappropriate heterochromatic 415 genes is not limited to fibroblasts. In contrast, there was no substantial chromatin 416 decompaction of the EDC in RIS keratinocytes (Fig. 6c). This reinforces the link 417 between aberrant expression of inappropriate genes and their decompaction.

419 Importantly, the decompacted chromatin state was not detected in hES cells for 420 either the EDC or DCC loci (Fig. 6c), where chromatin in general tends to be more 421 'open', exemplified by the DCC region (Fig. 6b) ${ }^{48,49}$. Therefore, senescence- 
associated decompaction of these heterochromatic loci is a distinct phenotype from

423 the 'open' chromatin configuration often seen in ES cells.

424

425 Multiple mechanisms might be involved in decompaction of $\mathrm{H} 3 \mathrm{~K} 9 \mathrm{me} 3$ regions

426 In previous Hi-C studies, an increase in long-range interaction between $\mathrm{H} 3 \mathrm{~K} 9 \mathrm{me} 3$

427 regions during senescence has been correlated with the detachment of the

$428 \mathrm{H} 3 \mathrm{~K} 9 \mathrm{me} 3$ regions from nuclear lamina, reduced interaction within the H3K9me3

429 regions, and SAHF formation (clustering of the H3K9me3 regions) ${ }^{25,33,50,51}$.

430 Consistently, in our recently generated in situ $\mathrm{Hi}-\mathrm{C}$ data in proliferative and RIS

431 IMR90 cells ${ }^{52}$, we observed that increased chromatin contacts during RIS tended to

432 be longer-range compared to decreased contacts, which were mostly short distance

433 (Extended Data Fig. 5a, b). We further characterized RIS-associated dynamic

434 chromatin interactions focusing on $\mathrm{H} 3 \mathrm{~K} 9 \mathrm{me} 3$ loci. In marked contrast to the features

435 of $\mathrm{H} 3 \mathrm{~K} 9 \mathrm{me} 3$ regions that are potentially involved in SAHFs (i.e., increased inter-

436 region contacts, decreased intra-region contacts, and reduced Lamin B1

437 occupancy) ${ }^{14,25,50}$, group 3 H3K9me3 peaks (defined in Fig. 4) exhibited the weakest

438 interaction increase with other $\mathrm{H} 3 \mathrm{~K} 9 \mathrm{me} 3$ peaks (Fig. 7a, b). Interestingly, group 2 of

439 H3K9me3 peaks (permissive without increased gene expression) showed more

440 increased contacts than not only group 3 but also group 1 . The nature of group 2

$441 \mathrm{H} 3 \mathrm{~K} 9 \mathrm{me} 3$ peaks and their involvement in higher-order heterochromatin

442 reorganization during RIS is unclear. Notably, the reduction in Lamin B1 occupancy

443 in group 2, similar to group 1, was significantly stronger than group 3 (Fig. 4i); thus,

444 group 2 of $\mathrm{H} 3 \mathrm{~K} 9 \mathrm{me} 3$ regions might play some role in heterochromatin reorganization.

446 We next examined individual gene loci. For example, the EDC locus showed reduced

447 interaction with other H3K9me3 peaks (Fig. 7c, 'LCE2, IVL') but increased contacts

448 within the locus (Fig. 7d). We reanalyzed $\mathrm{Hi}-\mathrm{C}$ data generated in WI38 fibroblasts ${ }^{25}$

449 and found a similar alteration in the EDC locus during replicative senescence (RS, 
450 Fig. 7e). Hi-C mapping in human keratinocytes was recently published ${ }^{53}$. We 451 reanalyzed the data and compared them with the senescent fibroblasts data. In 452 keratinocytes, new loop structures were established within the EDC locus during 453 terminal differentiation (Fig. 7f, Extended Data Fig. 5c). Interestingly, the new 454 contacts in the EDC locus in RIS IMR90 and RS WI38 fibroblasts resembled the 455 loops identified in keratinocytes (Fig. 7d-f, arrows). Together with the FISH data (Fig. $4562 \mathrm{~b}, \mathrm{~g}$ ), this suggests that the EDC locus escaped from SAHFs and decompacted at 457 the peri-SAHF region, where it gained active 3D regulatory interactions. In contrast, $458 N L R P 3$, another gene that was derepressed from a H3K9me3 region, exhibited a 459 highly distinct pattern. NLRP3 is located near the border of a H3K9me3-rich 460 topologically associating domain (TAD) in IMR90 fibroblasts (Fig. 7g). TADs are 461 megabase-sized chromatin regions, within which genomic regions interact with each 462 other with high frequency ${ }^{54-57}$. These proximal chromatin contacts around NLRP3 463 were largely lost and thus the TAD structure was disrupted in fibroblasts (Fig. $7 \mathrm{~g}$, 464 arrows). NLRP3 is predominantly expressed in immune cells, such as macrophages, 465 and the encoded protein NLRP3 is a component of the NLRP3-inflammasome, a 466 critical mediator of innate immune responses ${ }^{58,59}$. In addition, the role of NLRP3 in 467 inflammatory SASP in fibroblast senescence has been demonstrated ${ }^{60}$. We 468 reanalyzed public $\mathrm{Hi}-\mathrm{C}$ and ChIP-seq datasets in THP1 cells, a spontaneously 469 immortalized human monocytic cell line. NLRP3 was located within an open region in 470 THP1 cells and the TAD disruption seen in RIS fibroblasts was not detected during 471 macrophage differentiation, which is accompanied by NLRP3 upregulation (Fig. $4727 \mathrm{~h})^{61,62}$, reinforcing the functional relevance of the unique mechanism for 473 senescence-specific activation of genes that are otherwise tightly silenced within 474 H3K9me3 regions. Interestingly, we recently identified a similar TAD disruption, in 475 which genes, best exemplified by NRG1 (encoding a SASP factor), can escape from 476 a H3K27me3 repressive 3D environment during $\mathrm{RIS}^{52}$. Indeed, ChIP-seq data 477 showed a reduction of H3K27me3 in this region (Extended Data Fig. 5d). Together, 
478 our data suggest that the mechanisms for decompaction of the lineage-inappropriate

479 genes within $\mathrm{H} 3 \mathrm{~K} 9 \mathrm{me} 3$ occupied regions might differ between gene loci.

481 A recent study reported induction of NIrp3 in synovial fibroblasts (SFs) in a mouse 482 arthritis model ${ }^{63}$ : SFs isolated from mouse paws exposed to a danger signal, 483 monosodium urate crystals (MSU), twice (MSU/MSU), but not once (-/MSU), 484 exhibited upregulation of NIrp3. Such a delayed alteration in gene expression is 485 reminiscent of a senescent-like phenotype. We reanalyzed their RNA-seq data 486 (Extended Data Fig. 5e) and confirmed the significant upregulation of NIrp3, 487 alongside that of cytokines that are associated with senescence. Moreover, 488 MSU/MSU SFs exhibited senescence features and significant induction of several 489 EDC genes, compared to -/MSU SFs. Notably, rodents lack the Lce2 sub-cluster, but 490 instead they have an expansion of the Lce1 genes ${ }^{64}$. Although epigenomic 491 characterization is required for further interpretation, this result suggests EDC genes and NIrp3 can be expressed in senescent fibroblasts in vivo.

493

494

\section{Discussion}

In this work, we provide evidence that senescence is associated with the disruption of the H3K9me3-mediated 'lineage barrier' at specific gene loci. This results in the 'leaky' expression of specific heterochromatic genes, which are otherwise tightly silenced. This process is accompanied by a localized decompaction and increased accessibility of chromatin, without apparent loss of H3K9me3. We show that the same genes can be induced via distinct mechanisms: the 'default' mechanism of orthotopic cell types and a senescent-associated mechanism in ectopic cell types. Notably, we demonstrate that the default mechanism appears to be dominant when those genes are induced in senescent orthotopic cells; therefore, the initial cell typespecific chromatin states are perhaps critical for how these genes are derepressed in response to stress. Considering that decompaction of those gene loci was closely 
correlated with SAHF formation, it might be an integrated part of high-order

507 chromatin structural reorganization, specifically occurring during senescence.

509 NLRP3-inflammasome has been implicated in aging and is considered a therapeutic 510 target against age-associated inflammatory diseases ${ }^{65,66}$. Targeting senescent cells 511 is an emerging anti-aging therapeutics approach, including 'senolytics' (drugs that 512 selectively kill senescent cells) or 'senomorphics' (drugs that suppress the SASP or 513 any other adverse functionality of senescent cells ${ }^{67}$. Our data suggest that NLRP3 514 might be a potential senomorphic target ${ }^{59,60}$. Currently it is not clear whether the other 515 genes identified in this study are involved in the senescence phenotype. However, it 516 was shown that LCE1 is a downstream target of p53 in cancer cell lines and inhibits 517 the arginine methyltransferase, PRMT5, which is often upregulated in various 518 cancers and a potential therapeutic target ${ }^{68}$. PRMT5 was also shown to have anti519 senescence activity in osteosarcoma cells ${ }^{69}$. While ectopic LCE2A limited cell 520 proliferation (Extended Data Fig. 1d, e), it remains to be elucidated whether or not 521 LCE2 inhibits PRMT5 in senescent fibroblasts. DCC, ZNF667, and PDPN have also 522 been implicated in cancer and rheumatoid arthritis ${ }^{70}$. For example, Netrin 1 receptor, 523 encoded by $D C C$, is involved in axon guidance and DCC has been identified as a 524 tumor suppressor gene in ectopic tissues, including colon and rectum ${ }^{71,72}$. A similar 525 aberrant derepression mechanism might apply in other pathological contexts, such 526 as early tumorigenesis and chronic inflammation.

\section{Materials and Methods}

\section{Cell culture}

530 Human diploid fibroblasts (HDFs) (IMR90, WI38 and BJ) were cultured in phenol-red531 free DMEM with 10\% FBS under physiological $5 \% \mathrm{O}_{2}$, except for population doubling 532 and colony formation assays, which were conducted in atmospheric $\mathrm{O}_{2}{ }^{19,37}$. RIS was 533 induced by retrovirus-mediated expression of either HRAS ${ }^{G 12 V}$ or HRAS ${ }^{G 12 V}$ fused to 
534 the estrogen receptor (ER) ligand-binding domain (ER:RAS) system in HDFs. Acute

535 DNA damage (acDD) was induced by treating cells with $100 \mu \mathrm{M}$ etoposide for 24

536 hours. To obtain DNA damage induced senescence (DDIS) cells, cells were treated

537 with $100 \mu \mathrm{M}$ etoposide for 48 hours and maintained for an additional five days in 538 drug-free media. To generate RIS-bypassed cells, HRAS ${ }^{\mathrm{G} 12 \mathrm{~V}}$ and E1A (adenoviral

539 oncoprotein) were co-transduced using the retroviral gene expression system $540(E 1 A+R A S)^{18}$. For population-doubling analysis, cells were counted and $2 \times 10^{6}$ cells 541 were plated on $10 \mathrm{~cm}$ plates every 3-4 days. For colony formation assays, 10,000 542 cells were plated on $35 \mathrm{~mm}$ plates and maintained for 30 days. Cells were fixed with $5434 \%$ formaldehyde and stained with Crystal Violet (Sigma). Dye intensity was 544 quantified as integrated density using "Fiji" image processing package.

546 Primary normal human keratinocytes were isolated from normal human skin. All 547 procedures involving human subjects were approved by the ethics committee of the 548 Ehime University Graduate School of Medicine, Toon, Ehime, Japan, and all subjects 549 provided written informed consent. The keratinocytes were cultured in Epilife medium 550 (Gibco) supplemented with human keratinocyte growth supplement (HKGS, Gibco) 551 under $21 \%$ atmospheric $\mathrm{O}_{2}$. To induce differentiation in culture, keratinocytes were 552 treated with $500 \mathrm{nM}$ phorbol 12-myristate 13-acetate (PMA, Sigma, P8139) for $48 \mathrm{~h}$ 553 after cells became confluent. RIS was induced by lentivirus-mediated expression of 554 HRAS $^{\mathrm{G} 12 \mathrm{~V}}$ in human keratinocytes.

555

556 The WA09/H9 human embryonic stem cells were obtained from WiCell, and cultured 557 in TeSR-E8 (StemCell Technologies \#05990) on Vitronectin-coated glass coverslips $558\left(0.5 \mu \mathrm{g} / \mathrm{cm}^{2}\right.$; ThermoFisher Scientific, A14700) in an atmosphere of $5 \% \mathrm{O}_{2} \& 5 \% \mathrm{CO}_{2}$ 559 at $37^{\circ} \mathrm{C}$ for $\mathrm{FISH}$ experiments. 
562 The following retroviral vectors were used ${ }^{19,34}$ : pLNCX2 (Clontech) for ER:HRAS ${ }^{\mathrm{G} 12 \mathrm{~V}}$; 563 pBabe-puro for HRAS ${ }^{G 12 V}$; pWZL-hygro for HRAS ${ }^{G 12 V}, p 16, E 1 A, p L P C$ for mVenus-

564 LCE2A, pMSCV-puro for miR30 sh-p53 (target sequence 5'565 CACTACAACTACATGTGTA-3') ${ }^{18}$, miR30 shHMGA1 (target sequence 5'566 ATGAGACGAAATGCTGATGTAT-3') and miR30 control $^{37}$, pSuper shCEBPB (gift 567 from Daniel Peeper $)^{40}$. The following lentiviral vectors were used: pLenti HRAS ${ }^{\mathrm{G} 12 \mathrm{~V}}$ 568 (gift from Eric Campeau, Addgene plasmid \# 22259).

569

$570 \quad$ Immunoblotting

571 Immunoblotting was performed as previously described ${ }^{19}$. Quantification of signal 572 intensity was performed using "Fiji" image processing package. Signal intensity of 573 targeted proteins were normalized to $\beta$ Actin.

574

575 Antibodies

576 The following antibodies were used for immunoblotting: anti-ßActin (Sigma, A5441, 577 1:5000), anti-Cyclin A (Sigma, C4170, 1:1000), anti-Involucrin (Sigma, I9018, 1:1000; 578 Santa Cruz, A-5, 1:1000), anti-p16 (H-156, Santa Cruz, sc-759, 1:500) and (G175579 1239, BD Pharmingen, 554079, 1:500), anti-p21 (F-5, Santa Cruz, sc-6246, 1:500), 580 anti-p53 (DO-1, Sigma, P6874, 1:1000), anti-C/EBPß (C-19, Santa Cruz, sc-150, 581 1:500), anti-H-Ras (F235, Santa Cruz, sc-29, 1:500), anti-E1A (Santa Cruz, sc-430, 582 1:2000), anti-HMGA1 (Active Motif, 39615, 1:2000). The anti-Pan-LCE2 antibody 583 (1:1000) was raised in rabbits against a synthetic peptide (RPRLFHRRRHQSPD), as 584 previously described (note, this antigen sequence perfectly matches human LCE2B, $585 \mathrm{C}$ and $\mathrm{D}$, and differs by one amino acid for LCE2A $)^{26}$. The following antibodies were 586 used for ChIP: anti-H3K9me3 (clone CMA318), anti-H3K27me3 (clone CMA323), 587 anti-H3K27ac (clone CMA309) ${ }^{73}$, C/EBPß (C-19, Santa Cruz, sc-150) ${ }^{20}$. 588 589 
590 RNA was prepared using the Qiagen RNeasy plus kit (Qiagen) according to the 591 manufacturer's instructions and reverse-transcribed to cDNA using the reverse 592 transcriptase Superscript III kit or High-Capacity cDNA Reverse Transcription Kit 593 (Life Technologies). qRT-PCR was performed with SYBR Green Master Mix (Takara) 594 or Fast SYBR ${ }^{\mathrm{TM}}$ Green Master Mix (Applied Biosystems). Data were normalized to 595 ACTB (encoding $\beta$ Actin) expression. The following primers were used ${ }^{21}$ :

596 ACTB forward: 5'-CGAGCACAGAGCCTCGCCTT-3'

597 ACTB reverse: 5'-CATCATCCATGGTGAGCTGGCGG-3'

598 DCC forward: 5'-AGCAGGAAGAAGTCAGTCAGTG-3'

599 DCC reverse: 5'-CTGTGGGGATGGTTCTGCTT-3'

600 IL1A forward: 5'-AACCAGTGCTGCTGAAGGA-3'

601 IL1A reverse: 5'-TTCTTAGTGCCGTGAGTTTCC-3'

602 IL6 forward: 5'-TGAAAGCAGCAAAGAGGCACTG-3'

603 IL6 reverse: 5'-TGAATCCAGATTGGAAGCATCC-3'

604 IL8 forward: 5'-AAGGAAAACTGGGTGCAGAG-3'

605 IL8 reverse: 5'-ATTGCATCTGGCAACCCTAC-3'

606 LCE2A forward: 5'-GGACCTGTCCCAGAGTGATG-3'

607 LCE2A reverse: 5'-GATCCAGGATGGGCTCTTG-3'

608 LCE2B forward: 5'-GGTTGACTAAACTCTGCCAGG-3'

609 LCE2B reverse: 5'-CACTGGGGCAGGCATTTA-3'

610 LCE2C forward: 5'-CTTGGGACTGAATGGCCAAG-3'

611 LCE2C reverse: 5'-GACTTGCAATTGGGGTGTTAC-3'

612 LCE2D forward: 5'-CTGCAGAAGAGCTCTGGTACTG-3'

613 LCE2D reverse: 5'-CTCCATCAAGCACAAAGTTCTG-3'.

614

615 DNA FISH

616 DNA FISH was performed as previously described ${ }^{14}$. Cells were plated onto glass 617 coverslips the day before fixation. Cells were pre-treated with digitonin/CSK buffer 
$618(150 \mathrm{mg} / \mathrm{ml})$, then fixed in $4 \%$ paraformaldehyde. Cells were permeabilized with $0.2 \%$

$619(\mathrm{v} / \mathrm{v})$ Triton $\mathrm{X}-100$ in PBS, soaked in liquid nitrogen, treated in $0.1 \mathrm{M} \mathrm{HCl}$ and

620 dehydrated in $\mathrm{EtOH}$. Several genomic loci were observed by tiling the following

621 BAC/PAC probes along the EDC locus: RP6-121F19 (or RP11-81P11), RP11-655C3,

622 RP11-709L1, RP11-157C12, RP11-352B17, RP11-766D13 and RP11-157A11; OR

623 locus: RP11-643N11, RP11-344I22, RP11-643G14 and RP11-1084O3, or DCC

624 locus: RP11-25O3, RP11-108B6, RP11-1077M23, RP11-315H18, RP11-933N21 and

625 RP11-186B13; TMEM132B locus: RP11-433N22, RP11-1139A9, RP11-728C18 and

626 RP11-626N23; NLRP3 locus: RP11-243D8, RP11-951B14, RP11-908P10, RP11-

627121022 and RP11-248A15. Fluorescent directly labeled probes were generated

628 using a Nick Translation kit (Abbott) and/or purchased from Empire Genomics.

629 Confocal images were obtained using a Leica TCS SP5 microscope. The maximum

630 width of multi-probed signals (any colour) on the locus were measured on Leica

631 Application Suite Advanced Fluorescence (LAS-AF) software (Leica, Mannheim). For

$6323 \mathrm{D}$ images, confocal Z-stack images were projected to show $\mathrm{X}-\mathrm{Y}$ images. $\mathrm{X}-\mathrm{Z}$

633 images were generated by re-slicing and projection of 3D reconstructed images

634 using ImageJ. SADS were observed as previously described ${ }^{15}$ using an oligo against

635 the alpha-satellite repeat (5'-

636 CTTTTGATAGAgCAGTTTTGAAACACTCTTTTTGTAGAATCTGCAAGTGGATATTT

637 GG-3').

638

\section{Transcriptomic analysis}

640 RNA-seq was performed as previously described ${ }^{10}$. RNA was purified using the 641 Qiagen RNeasy plus kit according to the manufacturer's instructions. The quality was 642 checked using the Bioanalyzer with an Agilent RNA 6000 nano kit (Agilent) or Agilent 643 High Sensitivity RNA ScreenTape System. Libraries were prepared from six 644 biological replicates of each condition using TruSeq Stranded mRNA Library Prep Kit 645 or Illumina Stranded mRNA Prep Kit (Illumina) according to the manufacturer's 
646 instructions and sequenced using the HiSeq-4000 and Novaseq-6000 platforms

647 (Illumina). Low quality reads (quality score < 20) were removed from each sample

648 using Cutadapt $(1.10 .0)^{74}$. Reads were mapped to the human reference genome

649 (hg19) using the STAR aligner ${ }^{75}$. Read counting was carried out using Rsubread ${ }^{76}$

650 and differentially expressed genes, comparing RIS and growing conditions, were

651 identified using edge ${ }^{77}$. For heatmap analyses, raw counts were TMM normalized

652 and converted to transcripts-per-million (TPM). The EDC locus was defined as

653 Chr1:152,056,619-Chr1:153,234,364. The EDC gene list was retrieved from the

654 UCSC Table Browser ${ }^{78}$.

655

656 For microarray data analysis, data previously deposited in NCBI GEO as image 657 corrected, quantile normalised and log2 transformed intensities were downloaded 658 using the Bioconductor GEOquery package ${ }^{79}$. A linear model was fitted on the 659 resulting values and log2-fold changes for each of the desired contrasts was 660 computed using a simple empirical Bayes model with functions of the limma 661 package ${ }^{80}$.

662

663 To determine the tissue specificity of a gene, we used the Human Protein Atlas 664 (HPA) resource (https://www.proteinatlas.org//) ${ }^{45}$ and its associated criteria for 665 defining the tissue enhanced/enriched status of gene (used for Fig. 5a). In order to 666 estimate a tissue specificity score for each gene in different gene groups (such as in 667 Extended Data Fig. 4a), we used the transcriptomic data from the HPA and 668 computed the Tau score, which was shown previously ${ }^{81}$ to be the best metric for 669 computing tissue specificity. The score ranges from 0 to 1 , with 0 indicating a 670 ubiquitously expressed gene and 1 a highly tissue specific one.

671

672 ChIP-seq and ATAC-seq analysis 
673 ChIP-seq and ATAC-seq were performed as previously described ${ }^{10}$. Low quality

674 reads (quality score $<20$ ) were removed from each sample using Cutadapt $(1.10 .0)^{74}$.

675 Reads were mapped to the human reference genome (hg19) using BWA (v0.7.12) ${ }^{82}$

676 and reads mapping to the "blacklisted" regions identified by ENCODE ${ }^{83}$ were

677 removed from further analysis. H3K27ac and ATAC-seq peaks were called with

$678 \mathrm{MACS}^{84}$ and $\mathrm{H} 3 \mathrm{~K} 9 \mathrm{me} 3$ enriched domains were identified using $\mathrm{EDD}^{85}$. A high

679 confidence peak set was identified for data types and conditions separately keeping

680 only those regions that were present in at least two replicates. These intersections as

681 well as the overlaps between H3K27ac, H3K9me3 and ATAC-seq high confidence

682 peak sets were then identified using bedtools $(\mathrm{v} 2.26 .0)^{86}$. Average read coverage on

683 the EDC locus was summarized on $1 \mathrm{~kb}$ long bins from $\mathrm{THOR}^{87}$ normalized coverage

684 files (TMM normalization) using the deepTools package ${ }^{88}$, which was also used to

685 plot heatmaps of the THOR normalized signal. The same THOR normalized

686 coverage files were loaded and visualized in the genome browser.

687

688 Hi-C analysis

$689 \mathrm{Hi}-\mathrm{C}$ libraries were aligned using $\mathrm{HiC}-\mathrm{Pro}^{89}$ against the hg19 genome after initial QC

690 checks using FastQC (https://www.bioinformatics.babraham.ac.uk/projects/fastqc/).

691 Significant differential interactions, as well as distance corrected ICE-normalized $\mathrm{Hi}-\mathrm{C}$

692 maps, were estimated using diffHiC ${ }^{90}$. Visualisation of distance-corrected and ICE

693 normalized ${ }^{91} \mathrm{Hi}-\mathrm{C}$ matrices and associated annotation (arcs representing significant

694 interactions) was performed using $\mathrm{HiCvizR}^{52}$.

695

696 Data availability

697 Human keratinocytes RNA-seq and ChIP-seq data and IMR90 ATAC-seq data (RIS)

698 and RNA-seq data generated for this study have been deposited at GEO

699 (GSE130457, GSE180469). Control ATAC-seq data were previously published

$700(\text { GSE103590 })^{10}$. Microarray datasets (Extended Data Fig. 1) were previously 
701 published (GSE53379 and GSE59522) ${ }^{18,19}$. RNA-seq data for RIS/DDIS IMR90 cells

702 are available at GSE $72407^{20}$. Other senescence RNA-seq data ${ }^{23,24}$ were downloaded

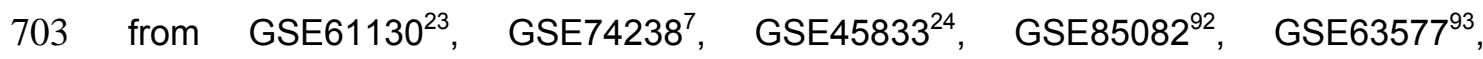
704 GSE56293 ${ }^{94}$, GSE130306 ${ }^{25}$. SFs RNA-seq were downloaded from GSE16374963. 705 HPA RNA-seq data were downloaded from The Human Protein Atlas (HPA) 706 (https://www.proteinatlas.org) E-MTAB-2836 ${ }^{45}$. THP1 RNA-seq data were 707 downloaded from GSE9680062. ChIP-seq for H3K9m3, H3K27me3 ${ }^{12}, \mathrm{H} 3 \mathrm{~K} 27 \mathrm{ac}{ }^{10}$, 708 and Lamin $B 1^{14}$ are available at GSE38448, GSE103590, and GSE49341, 709 respectively. ChIP-seq data for hES cells were downloaded from ENCODE, 710 GSE29611 ${ }^{83}$. H3K27ac ChIP-seq and ATAC-seq data for THP1 cells were 711 downloaded from the Gene Expression Omnibus (GEO) accession number 712 GSE9680062. Hi-C data (IMR90 cells) can be found at GSE135090. Hi-C data 713 (human keratinocytes) were downloaded from GSE84660. Hi-C data (THP1 cells) 714 were downloaded from Sequence Read Archive accession number PRJNA385337 ${ }^{62}$. $715 \mathrm{Hi}-\mathrm{C}$ data (WI38 cells) were downloaded from GSE130306 ${ }^{25}$.

\section{Acknowledgements}

718 We thank all members of the Narita laboratory for helpful discussions, A. Young for 719 editing, and staff of the CRUK-Cl core facilities (Microscopy, Genomics, Biorepository, 720 Bioinformatics and Research Instrumentation) for technical support, T. Tsuda and E. 721 Nishihama at the Ehime University for isolation and expansion of human primary 722 keratinocytes. This work is supported by a Cancer Research UK Cambridge Institute 723 Core Grant (C9545/A29580) to the M.N. laboratory, and in part by the Human 724 Frontier Science Program (RGY0078/2010) (MN). M.N. is also supported by the 725 Medical Research Council (MR/M013049/1), BBSRC (BB/S013466/1) and BIRAX 726 Ageing (65BX18MNIB). I.O. is supported by the Cancer Research UK Pioneer Grant 727 (C63389/A30462). S.A.S and D.B. are supported by the Medical Research Council 728 (MC_UU_12022/10). S.S. is supported by the UKRI Biotechnology and Biological 
Science Research Council (BB/J004480/1), the UKRI Medical Research Council

730 (MR/T016787/1), and a Career Progression Fellowship from the Babraham Institute.

731 P.J.R.-G. is supported by the BBSRC (BBS/E/B/000C0422). H. Kimura is supported

732 by JSPS KAKENHI (JP17H01417 and JP18H05527).

733

734 Author Contributions: Masashi N., S.A.S. and K.T. conceived the project. K.T., A.J.P., 735 S.S., Y.I., K.K., C.B.R. Masako N. T.S. H. Kugoh, P.J.R.-G., P.F. and Masashi N. 736 designed and performed experiments, and analyzed data. D.B., I.O., G.St.C.S., 737 A.S.L.C. and S.A.S analyzed sequencing data. I.O. analyzed Hi-C data. H. Kimura 738 provided histone antibodies. K. Shiraishi and K Sayama provided the human 739 keratinocytes. Masashi N. and K.T. wrote the manuscript with inputs from all authors.

740

741

742

743

744

745

746

747

748

749

750

751

752

753

754

755

756

757

\section{Competing interests}

P.F. and S.S. are co-founders Enhanc3D Genomics Ltd. All other authors declare no competing interests.

\section{References}

1. Gorgoulis, V. et al. Cellular Senescence: Defining a Path Forward. Cell 179, 813827 (2019).

2. Faget, D. V., Ren, Q. \& Stewart, S. A. Unmasking senescence: context-dependent effects of SASP in cancer. Nat Rev Cancer 19, 439-453 (2019).

3. Coppé, J.-P., Desprez, P.-Y., Krtolica, A. \& Campisi, J. The SenescenceAssociated Secretory Phenotype: The Dark Side of Tumor Suppression. Annu Rev Pathology Mech Dis 5, 99-118 (2010).

4. Chan, A. S. L. \& Narita, M. Short-term gain, long-term pain: the senescence life cycle and cancer. Gene Dev 33, 127-143 (2019).

5. Rai, T. S. \& Adams, P. D. Lessons from senescence: Chromatin maintenance in non-proliferating cells. Biochim Biophys Acta 1819, 322-31 (2011). 
6. Nacarelli, T., Liu, P. \& Zhang, R. Epigenetic Basis of Cellular Senescence and Its Implications in Aging. Genes-basel 8, 343 (2017).

7. Tasdemir, N. et al. BRD4 Connects Enhancer Remodeling to Senescence Immune Surveillance. Cancer Discov 6, 612-629 (2016).

8. Sen, P. et al. Histone Acetyltransferase p300 Induces De Novo Super-Enhancers to Drive Cellular Senescence. Mol Cell 73, 684-698.e8 (2019).

9. Cecco, M. et al. Genomes of replicatively senescent cells undergo global epigenetic changes leading to gene silencing and activation of transposable elements. Aging Cell 12, 247-256 (2013).

10. Parry, A. J. et al. NOTCH-mediated non-cell autonomous regulation of chromatin structure during senescence. Nat Commun 9, 1840 (2018).

11. Chicas, A. et al. H3K4 demethylation by Jarid1a and Jarid1b contributes to retinoblastoma-mediated gene silencing during cellular senescence. Proc National Acad Sci 109, 8971-8976 (2012).

12. Chandra, T. et al. Independence of Repressive Histone Marks and Chromatin Compaction during Senescent Heterochromatic Layer Formation. Mol Cell 47, 203214 (2012).

13. Shah, P. P. et al. Lamin B1 depletion in senescent cells triggers large-scale changes in gene expression and the chromatin landscape. Gene Dev 27, 1787-99 (2013).

14. Sadaie, M. et al. Redistribution of the Lamin B1 genomic binding profile affects rearrangement of heterochromatic domains and SAHF formation during senescence. Gene Dev 27, 1800-1808 (2013).

15. Swanson, E. C., Manning, B., Zhang, H. \& Lawrence, J. B. Higher-order unfolding of satellite heterochromatin is a consistent and early event in cell senescence. J Cell Biol 203, 929-942 (2013).

16. Dambacher, S., Hahn, M. \& Schotta, G. Epigenetic regulation of development by histone lysine methylation. Heredity 105, 24-37 (2010).

17. Becker, J. S., Nicetto, D. \& Zaret, K. S. H3K9me3-Dependent Heterochromatin: Barrier to Cell Fate Changes. Trends Genet 32, 29-41 (2016).

18. Kirschner, K. et al. Phenotype Specific Analyses Reveal Distinct Regulatory Mechanism for Chronically Activated p53. Plos Genet 11, e1005053 (2015).

19. Young, A. R. J. et al. Autophagy mediates the mitotic senescence transition. Gene Dev 23, 798-803 (2009).

20. Hoare, M. et al. NOTCH1 mediates a switch between two distinct secretomes during senescence. Nat Cell Biol 18, 979-992 (2016). 
21. Jackson, B. et al. Late Cornified Envelope Family in Differentiating EpitheliaResponse to Calcium and Ultraviolet Irradiation. J Invest Dermatol 124, 1062-1070 (2005).

22. Niehues, H. et al. Late cornified envelope (LCE) proteins: distinct expression patterns of LCE2 and LCE3 members suggest nonredundant roles in human epidermis and other epithelia. Br J Dermatology 174, 795-802 (2016).

23. Herranz, N. et al. mTOR regulates MAPKAPK2 translation to control the senescence-associated secretory phenotype. Nat Cell Biol 17, 1205-1217 (2015).

24. Loayza-Puch, F. et al. p53 induces transcriptional and translational programs to suppress cell proliferation and growth. Genome Biol 14, R32 (2013).

25. Sati, S. et al. 4D Genome Rewiring during Oncogene-Induced and Replicative Senescence. Mol Cell 78, 522-538.e9 (2020).

26. Bergboer, J. G. M. et al. Psoriasis Risk Genes of the Late Cornified Envelope-3 Group Are Distinctly Expressed Compared with Genes of Other LCE Groups. Am J Pathology 178, 1470-1477 (2011).

27. Buenrostro, J. D., Giresi, P. G., Zaba, L. C., Chang, H. Y. \& Greenleaf, W. J. Transposition of native chromatin for fast and sensitive epigenomic profiling of open chromatin, DNA-binding proteins and nucleosome position. Nat Methods 10, 1213$1218(2013)$.

28. Creyghton, M. P. et al. Histone $\mathrm{H} 3 \mathrm{~K} 27$ ac separates active from poised enhancers and predicts developmental state. Proc National Acad Sci 107, 21931-21936 (2010).

29. Malnic, B., Godfrey, P. A. \& Buck, L. B. The human olfactory receptor gene family. Proc National Acad Sci 101, 2584-2589 (2004).

30. Deng, Z. et al. Late Cornified Envelope Group I, a Novel Target of p53, Regulates PRMT5 Activity. Neoplasia 16, 656-664 (2014).

31. Ezhkova, E. et al. Ezh2 Orchestrates Gene Expression for the Stepwise Differentiation of Tissue-Specific Stem Cells. Cell 136, 1122-1135 (2009).

32. Wurm, S. et al. Terminal epidermal differentiation is regulated by the interaction of Fra-2/AP-1 with Ezh2 and ERK1/2. Gene Dev 29, 144-56 (2015).

33. Criscione, S. W., Teo, Y. V. \& Neretti, N. The Chromatin Landscape of Cellular Senescence. Trends Genetics Tig 32, 751-761 (2016).

34. Narita, M. et al. Rb-Mediated Heterochromatin Formation and Silencing of E2F Target Genes during Cellular Senescence. Cell 113, 703-716 (2003).

35. Mardaryev, A. N. et al. p63 and Brg1 control developmentally regulated higherorder chromatin remodelling at the epidermal differentiation complex locus in epidermal progenitor cells. Development 141, 101-111 (2014).

36. Chandra, T. \& Narita, M. High-order chromatin structure and the epigenome in SAHFs. Nucleus 4, 23-28 (2013). 
832 37. Narita, M. et al. A Novel Role for High-Mobility Group A Proteins in Cellular

833 Senescence and Heterochromatin Formation. Cell 126, 503-514 (2006).

834 38. Shimi, T. et al. The role of nuclear lamin B1 in cell proliferation and senescence.

835 Gene Dev 25, 2579-93 (2011).

836 39. Rodier, F. et al. Persistent DNA damage signalling triggers senescence-

837 associated inflammatory cytokine secretion. Nat Cell Biol 11, 973-979 (2009).

838 40. Kuilman, T. et al. Oncogene-Induced Senescence Relayed by an Interleukin-

839 Dependent Inflammatory Network. Cell 133, 1019-1031 (2008).

840 41. Thurman, R. E. et al. The accessible chromatin landscape of the human genome.

$841 \quad$ Nature 489, 75-82 (2012).

842 42. Stergachis, A. B. et al. Developmental fate and cellular maturity encoded in

843 human regulatory DNA landscapes. Cell 154, 888-903 (2013).

844 43. Heinz, S., Romanoski, C. E., Benner, C. \& Glass, C. K. The selection and

845 function of cell type-specific enhancers. Nat Rev Mol Cell Bio 16, 144154 (2015).

846 44. Jordan, I. K., Rogozin, I. B., Glazko, G. V. \& Koonin, E. V. Origin of a substantial 847 fraction of human regulatory sequences from transposable elements. Trends Genet

848 19, 68-72 (2003).

849 45. Uhlén, M. et al. Proteomics. Tissue-based map of the human proteome. Sci New 850 York N Y 347, 1260419 (2015).

851 46. Song, X. et al. Autophagy deficient keratinocytes display increased DNA damage, 852 senescence and aberrant lipid composition after oxidative stress in vitro and in vivo.

853 Redox Biol 11, 219-230 (2017).

854 47. Lin, A. W. \& Lowe, S. W. Oncogenic ras activates the ARF-p53 pathway to

855 suppress epithelial cell transformation. Proc National Acad Sci 98, 5025-5030 (2001).

856 48. Gaspar-Maia, A., Alajem, A., Meshorer, E. \& Ramalho-Santos, M. Open

857 chromatin in pluripotency and reprogramming. Nat Rev Mol Cell Biology 12, 36-47

858 (2011).

859 49. Meshorer, E. \& Misteli, T. Chromatin in pluripotent embryonic stem cells and

860 differentiation. Nat Rev Mol Cell Bio 7, 540-546 (2006).

861 50. Chandra, T. et al. Global Reorganization of the Nuclear Landscape in Senescent 862 Cells. Cell Reports 10, 471-483 (2015).

863 51. Iwasaki, O. et al. Involvement of condensin in cellular senescence through gene 864 regulation and compartmental reorganization. Nat Commun 10, 5688 (2019).

865 52. Olan, I. et al. Transcription-driven cohesin repositioning rewires chromatin loops

866 in cellular senescence. Biorxiv 823831 (2019) doi:10.1101/823831. 
867

868

869

870

871

872

873

874

875

876

877

878

879

880

881

882

883

884

885

886

887

888

889

890

891

892

893

894

895

896

897

898

899

900

901

902

53. Rubin, A. J. et al. Lineage-specific dynamic and pre-established enhancerpromoter contacts cooperate in terminal differentiation. Nat Genet 49, 1522-1528 (2017).

54. Schoenfelder, S. \& Fraser, P. Long-range enhancer-promoter contacts in gene expression control. Nat Rev Genetics 20, 437-455 (2019).

55. Dixon, J. R. et al. Topological domains in mammalian genomes identified by analysis of chromatin interactions. Nature 485, 376-80 (2013).

56. Nora, E. P. et al. Spatial partitioning of the regulatory landscape of the Xinactivation centre. Nature 485, 381-5 (2012).

57. Sexton, T. et al. Three-dimensional folding and functional organization principles of the Drosophila genome. Cell 148, 458-72 (2012).

58. Swanson, K. V., Deng, M. \& Ting, J. P.-Y. The NLRP3 inflammasome: molecular activation and regulation to therapeutics. Nat Rev Immunol 19, 477-489 (2019).

59. Chandra, S., Ehrlich, K. C., Lacey, M., Baribault, C. \& Ehrlich, M. Epigenetics and expression of key genes associated with cardiac fibrosis: NLRP3, MMP2, MMP9, CCN2/CTGF and AGT. Epigenomics-uk 13, 219-234 (2021).

60. Acosta, J. C. et al. A complex secretory program orchestrated by the inflammasome controls paracrine senescence. Nat Cell Biol 15, 978-990 (2013).

61. Kong, F. et al. Curcumin Represses NLRP3 Inflammasome Activation via TLR4/MyD88/NF-KB and P2X7R Signaling in PMA-Induced Macrophages. Front Pharmacol 7, 369 (2016).

62. Phanstiel, D. H. et al. Static and Dynamic DNA Loops form AP-1-Bound Activation Hubs during Macrophage Development. Mol Cell 67, 1037-1048.e6 (2017).

63. Friščić, J. et al. The complement system drives local inflammatory tissue priming by metabolic reprogramming of synovial fibroblasts. Immunity 54, 1002-1021.e10 (2021).

64. Brown, S. J. et al. Rodent Lce Gene Clusters; New Nomenclature, Gene Organization, and Divergence of Human and Rodent Genes. J Invest Dermatol 127, 1782-1786 (2007).

65. Stojanović, S. D., Fiedler, J., Bauersachs, J., Thum, T. \& Sedding, D. G. Senescence-induced inflammation: an important player and key therapeutic target in atherosclerosis. Eur Heart J 41, 2983-2996 (2020).

66. Liu, D. et al. Inflammageing in the cardiovascular system: mechanisms, emerging targets, and novel therapeutic strategies. Clin Sci 134, 2243-2262 (2020).

67. Robbins, P. D. et al. Senolytic Drugs: Reducing Senescent Cell Viability to Extend Health Span. Annu Rev Pharmacol 61, 1-25 (2020). 
903

904

905

906

907

908

909

910

911

912

913

914

915

916

917

918

919

920

921

922

923

924

925

926

927

928

929

930

931

932

933

934

935

936

937

938

68. Shailesh, H., Zakaria, Z. Z., Baiocchi, R. \& Sif, S. Protein arginine methyltransferase 5 (PRMT5) dysregulation in cancer. Oncotarget 9, 36705-36718 (2018).

69. Li, Y.-H. et al. PRMT5-TRIM21 interaction regulates the senescence of osteosarcoma cells by targeting the TXNIP/p21 axis. Aging 12, 2507-2529 (2020).

70. Croft, A. P. et al. Rheumatoid synovial fibroblasts differentiate into distinct subsets in the presence of cytokines and cartilage. Arthritis Res Ther 18, 270 (2016).

71. Castets, M. et al. DCC constrains tumour progression via its dependence receptor activity. Nature 482, 534-7 (2011).

72. Mehlen, P. \& Fearon, E. R. Role of the Dependence Receptor DCC in Colorectal Cancer Pathogenesis. J Clin Oncol 22, 3420-3428 (2004).

73. Kimura, H., Hayashi-Takanaka, Y., Goto, Y., Takizawa, N. \& Nozaki, N. The Organization of Histone H3 Modifications as Revealed by a Panel of Specific Monoclonal Antibodies. Cell Struct Funct 33, 61-73 (2008).

74. Martin, M. Cutadapt removes adapter sequences from high-throughput sequencing reads. Embnet J 17, 10-12 (2011).

75. Dobin, A. et al. STAR: ultrafast universal RNA-seq aligner. Bioinformatics 29, 1521 (2012).

76. Liao, Y., Smyth, G. K. \& Shi, W. The Subread aligner: fast, accurate and scalable read mapping by seed-and-vote. Nucleic Acids Res 41, e108 (2013).

77. Robinson, M. D., McCarthy, D. J. \& Smyth, G. K. edgeR: a Bioconductor package for differential expression analysis of digital gene expression data. Bioinformatics 26, 139-140 (2009).

78. Karolchik, D. et al. The UCSC Table Browser data retrieval tool. Nucleic Acids Res 32, 493D - 496 (2004).

79. Davis, S. \& Meltzer, P. S. GEOquery: a bridge between the Gene Expression Omnibus (GEO) and BioConductor. Bioinformatics 23, 1846-1847 (2007).

80. Ritchie, M. E. et al. limma powers differential expression analyses for RNAsequencing and microarray studies. Nucleic Acids Res 43, e47 (2015).

81. Kryuchkova-Mostacci, N. \& Robinson-Rechavi, M. A benchmark of gene expression tissue-specificity metrics. Brief Bioinform bbw008 (2016) doi:10.1093/bib/bbw008.

82. Li, H. \& Durbin, R. Fast and accurate short read alignment with Burrows-Wheeler transform. Bioinformatics 25, 1754-1760 (2009).

83. Dunham, I. et al. An integrated encyclopedia of DNA elements in the human genome. Nature 489, 57-74 (2012). 
84. Zhang, Y. et al. Model-based analysis of ChIP-Seq (MACS). Genome Biol 9,

940 R137 (2008).

941 85. Lund, E., Oldenburg, A. R. \& Collas, P. Enriched domain detector: a program for detection of wide genomic enrichment domains robust against local variations. Nucleic Acids Res 42, e92 (2014).

86. Quinlan, A. R. \& Hall, I. M. BEDTools: a flexible suite of utilities for comparing genomic features. Bioinform Oxf Engl 26, 841-2 (2010). (2016).

88. Ramírez, F. et al. deepTools2: a next generation web server for deep-sequencing data analysis. Nucleic Acids Res 44, W160-5 (2016).

951 89. Servant, N. et al. HiC-Pro: an optimized and flexible pipeline for $\mathrm{Hi}-\mathrm{C}$ data

952 processing. Genome Biol 16, 259 (2015).

953 90. Lun, A. T. L. \& Smyth, G. K. diffHic: a Bioconductor package to detect differential 954 genomic interactions in Hi-C data. Bmc Bioinformatics 16, 258 (2015).

955 91. Imakaev, M. et al. Iterative correction of $\mathrm{Hi}-\mathrm{C}$ data reveals hallmarks of 956 chromosome organization. Nat Methods 9, 999-1003 (2012).

957 92. Muniz, L. et al. Control of Gene Expression in Senescence through 958 Transcriptional Read-Through of Convergent Protein-Coding Genes. Cell Reports 21, 959 2433-2446 (2017).

960 93. Marthandan, S. et al. Similarities in Gene Expression Profiles during In Vitro 961 Aging of Primary Human Embryonic Lung and Foreskin Fibroblasts. Biomed Res Int 962 2015, 1-17 (2015).

963 94. Alspach, E. et al. p38MAPK Plays a Crucial Role in Stromal-Mediated 964 Tumorigenesis. Cancer Discov 4, 716-729 (2014). 
968 Figure 1. Aberrant expression of lineage-inappropriate LCE2 genes during senescence. (a) Comparative analysis of LCE2 expression by immunoblotting during fibroblast (IMR90) RIS (RAS-induced senescence) and keratinocyte (KC)

971 differentiation (Diff). RIS was induced by retroviral expression of HRAS-G12V. Prolif, 972 vector control proliferating cells. KC Diff was induced using PMA for 48h. Undiff, 973 undifferentiated control. IVL, Involucrin, is an EDC-encoded KC Diff marker.

974 Densitometry quantification was performed by ImageJ, normalized to BActin $(n \geq 3)$. $975{ }^{*} P<0.05,{ }^{* *} P<0.01,{ }^{* *} P<0.001$, unpaired t-test. (b) Representative genome 976 browser images, centred around the EDC of the cells indicated. ER:HRAS-G12V 977 expressing IMR90 cells were cultured with $4 \mathrm{OHT}$ (RIS) or without $4 \mathrm{OHT}$ (Prolif) for 6 978 days. ChIP-seq data were THOR-normalized. The region with the orange shading is 979 magnified in the right-hand panel. (c) Averages of THOR-normalized read counts for $980 \mathrm{H} 3 \mathrm{~K} 9 \mathrm{me} 3$ and H3K27me3 signals on the EDC locus of the cells indicated. ${ }^{* * *} P<$ $981 \quad 0.001$, t-test and Shapiro-Wilkes normality test. (d) Representative genome browser 982 images of ATAC-seq at the EDC and OR loci $(n \geq 2)$. The same ChIP-seq data are 983 used as in $\mathbf{b}$.

984 985 Figure 2. Decompaction of EDC locus in senescence IMR90 cells. (a) 986 Representative genome browser images of Lamin B1 ChIP-seq $(n=3)$ in proliferative 987 and RIS IMR90 cells, with the location of BAC probes used in this study shown as 988 green/purple blocks underneath. The same H3K9me3 ChIP-seq data as in Fig. 1 989 were used as the reference. (b) Confocal DNA-FISH images using multiple BAC 990 probes tiled across the EDC locus as shown in a. (c) Quantification of the FISH 991 signal length for the EDC locus; proliferating (Prolif), acute DNA damage (acDD), $992 \mathrm{E} 1 \mathrm{~A}$ and RAS expressing (E1A+RAS), RAS-induced senescence (RIS), DNA 993 damage-induced senescence (DDIS), replicative senescence (RS) IMR90 cells; 994 undifferentiated (Undiff) and differentiated (Diff) keratinocytes (KC). ${ }^{* * *} P<0.001$, 
995 Mann-Whitney-Wilcoxon test ( $n=150$ alleles from 3 biological replicates). Bars are

996 median with inter-quartile range. (d) Time series analysis of EDC signal length 997 following addition of $100 \mathrm{nM} 4 \mathrm{OHT}$ to ER:HRAS-G12V IMR90 cells. At least 150 998 alleles were counted from 3 biological replicates. ${ }^{* \star *} P<0.001$ (Mann-Whitney999 Wilcoxon test). Bars are median with interquartile range. (e) Representative 1000 immunoblotting of the time series experiments as in $\mathbf{d}$. Arrow indicates specific bands. $1001{ }^{* *} P<0.01,{ }^{* *} P<0.001$ (one-way ANOVA with Fisher's LSD test, $\mathrm{n}=3$ ) (f) 1002 Quantification of SAHF- and SADS-positive nuclei of the time series experiments as 1003 in d. Representative images of SAHF and SADS at d7 are shown. SADS are 1004 indicated by a-satellite targeted oligo hybridization (red). Values are means +/- S.E.M. $1005(\mathrm{n}=3) .{ }^{* * *} P<0.001$ compared with d0, one-way ANOVA with Tukey's multiple 1006 comparisons test. (g) DNA-FISH using single BAC probes for EDC and OR loci as 1007 depicted in Fig. 2a (green and purple bars underneath top panel). Percentages of 1008 those loci colocalized with SAHF were measured. ${ }^{* * *} P<0.001$ unpaired t-test $(\mathrm{n}=$ $10093)$.

1010

1011 Figure 3. Decompaction of EDC locus and senescence effectors. (a) 1012 Quantification of SAHF-positive cells in RIS or proliferating (Vector) IMR90 cells with 1013 shHMGA1 (+) or control miR30 vector (-). (b) Quantification of the FISH signal length 1014 for the EDC locus in the indicated cells. (c) RNA-seq analysis in the indicated 1015 comparison. (d) Immunoblotting for the indicated proteins. ${ }^{*} P<0.05,{ }^{* \star} P<0.01,{ }^{* \star *} P$ $1016<0.001$, (one-way ANOVA with Fisher's LSD test, $n=3$ ). (e) Left, immunoblotting for 1017 the indicated proteins in IMR90 cells expressing control retroviral vector (Prolif), p16 1018 (p16-Sen), and HRAS-G12V (RIS). Two representative replicates are shown. Right, 1019 corresponding RNA-seq analysis, showing log-fold change (logFC) in the indicated 1020 conditions compared to corresponding proliferative controls. p16-Sen $(n=3)$, DDIS $1021(n=8)$, RIS ( $n=8)$. (f) Quantification of BrdU incorporation and SAHF-positive cells. 1022 Values are means $+/-$ SD $(\mathrm{n}=3) .{ }^{* * *} P<0.001$, (one-way ANOVA with Fisher's LSD 
1023 test). (g) Quantification of the FISH signal length for the EDC locus in the indicated

1024 cells. ${ }^{* *} \mathrm{P}<0.001$, (ordinary one-way ANOVA with Fisher's LSD test, $\mathrm{n} \geq 80$ allele

1025 from 3 biological replicates). Bars are median with inter-quartile range. (h) RNA-seq

1026 analysis - normalized expression heatmap (log-counts per million) of EDC genes in

$1027 \mathrm{p} 16-$-induced senescence ( $\mathrm{p} 16-\mathrm{Sen})$, DNA damage-induced senescence (DDIS) and

1028 RAS-induced senescence (RIS) with corresponding proliferative controls. (I - j) Two

1029 representative immunoblots $(n \geq 5)$ Arrows indicate the three specific isoforms. (i)

1030 and qPCR analysis (j) in indicated cells. ER:RAS IMR90 cells expressing shp53 and

1031 shC/EBP $\beta$ with corresponding control vectors were treated with $4 \mathrm{OHT}$ (RAS) for 6

1032 days. Values are means $+/-\operatorname{SD}(\mathrm{n}=5) .{ }^{*} P<0.05,{ }^{* *} P<0.01$, ${ }^{* * *} P<0.001$, (one-way

1033 ANOVA with Fisher's LSD test). (k) IGV tracks of THOR-normalized ChIP-seq signal

1034 of p53, C/EBP 3 and H3K27ac in Proliferating (Prolif) and RIS samples. (I)

1035 Quantification of the FISH signal length for the EDC locus in the indicated cells. ${ }^{* * *} P$

$1036<0.001$, Mann-Whitney-Wilcoxon test ( $n \geq 150$ alleles from 3 biological replicates).

1037 n.s., not significant.

1038

1039 Figure 4. H3K9me3 peaks characterisation. (a) Classification of H3K9me3 peaks

1040 into non-permissive (group 1) and permissive peaks (with de novo ATAC-seq and

1041 H3K27ac peaks) with (group 3) and without up-regulated genes (group 2). (b-i)

1042 Distribution of peak widths (Mb) (b), gene density (per Mb) (c), AT\% (d), SINE

1043 frequency (e), Alu repeats frequency (f), Lamin B1 THOR-normalized ChIP-seq

1044 signal in proliferating cells (g), Lamin B1 THOR-normalized ChIP-seq signal in RIS

1045 cells (h), differential LaminB1 signal between RIS and proliferative cells (i). In group

10463 , H3K9me3-peaks associated with the genes of interest are highlighted (b-i). All box

1047 plots correspond to the median, $25^{\text {th }}$ to $75^{\text {th }}$ percentiles, and the whiskers correspond

1048 to the $10^{\text {th }}$ to $90^{\text {th }}$ percentiles. Each pairwise comparison between groups was

1049 effectuated with a two-sided Student's t-test, ${ }^{* * *} P<0.001,{ }^{* *} P<0.01,{ }^{*} P<0.05$. n.s.,

1050 not significant. 
1052 Figure 5. Aberrant expression of lineage-specific genes and chromatin 1053 decompaction during senescence. (a) Tissue-dependent expression pattern of RIS 1054 upregulated genes that have RIS-unique H3K27ac/ATAC-peaks (at TSS +/- $250 \mathrm{bp}$ ) 1055 within H3K9me3 domains. RNA-seq datasets from IMR90 for RIS and DDIS (GSE-

1056 72407) and Human Protein Atlas (HPA) RNA-seq for normal tissues (E-MTAB-2836)

1057 were used. TPM, Transcripts-Per-Million. (b-d) Chromatin states of three additional 1058 examples of upregulated genes from H3K9me3 regions during RIS. Representative 1059 genome browser images (b). Representative multiprobe DNA-FISH images for the 1060 NLRP3 locus (c). Either single or two alternate colors were used in the probe set 1061 shown in b (green/purple blocks, underneath main panel). Measurement of FISH 1062 signals using the multiple BAC probes represented in (d). ${ }^{* *} P<0.001$, Mann1063 Whitney-Wilcoxon test ( $\mathrm{n} \geq 150$ alleles from 3 biological replicates). Bars, median 1064 with interquartile range.

1065

1066 Figure 6. Distinct mode of expression of lineage-appropriate and lineage1067 inappropriate genes during RIS. (a) Heatmap of normalized expression values 1068 (log-counts per million) of the EDC genes from RNA-seq samples of proliferating and 1069 RAS-induced $(n=6)$ keratinocytes $(K C s)$, as well as undifferentiated $(n=8)$ and 1070 differentiated $(n=9)$ KCs. (b) Genome browser images of H3K9me3 ChIP-seq of the 1071 EDC and DCC loci from the cells indicated. The human ES (hES) cell data are from 1072 ENCODE. Some of the IMR90 and human primary keratinocyte (KC) images were 1073 replotted for comparisons using the same datasets as in Fig. 1b (EDC) and Fig. 5b 1074 (DCC). (c) DNA-FISH analysis of the DCC and EDC loci in the cells indicated. ${ }^{* * *} P<$ 1075 0.001, Mann-Whitney-Wilcoxon test ( $n \geq 150$ alleles from 3 biological replicates). 1076 Bars are median with inter-quartile range. (d) qRT-PCR for the indicated mRNA in 
1077 vector control and RIS KCs. Error bars indicate mean $+/-$ S.E.M. $(\mathrm{n}=9) .{ }^{* \star \star} P<0.001$,

$1078{ }^{*} P<0.05$ paired t-test.

1079

1080 Figure 7. Differential 3D behaviour of permissive H3K9me3 peaks. (a) Total

$1081 \mathrm{H} 3 \mathrm{~K} 9 \mathrm{me}-\mathrm{H} 3 \mathrm{~K} 9 \mathrm{me} 3$ interactions (connectivity) of the individual $200 \mathrm{~kb}$ bins (sum of

1082 the interactions with all other $200 \mathrm{~kb}$ bins within $\mathrm{H} 3 \mathrm{~K} 9 \mathrm{me} 3$ peaks) within each group

1083 of H3K9me3 peaks (the same three groups as in Fig. 4). (b) Differential H3K9me3-

1084 H3K9me3 interaction between proliferative and RIS conditions in each group. Log-

1085 fold changes (logFC) of increased (top, green) and decreased interactions (bottom,

1086 blue) in each peak with all other H3K9me3 peaks are shown. Each pairwise

1087 comparison is performed using two-sided Student's t-test with ${ }^{* * *} P<0.001$, ${ }^{* *} P<$

1088 0.01. n.s., not significant. Boxplots in (a) and (b) represent the median, 25th to 75th

1089 percentiles, whiskers: 10th to 90th percentiles. (c) Significant LogFC (green -

1090 positive, blue - negative) of all the $\mathrm{Hi}-\mathrm{C}$ interactions involving each of the H3K9me3

1091 peaks of interest within group 3 compared to the OR region (OR10T2) neighbouring

1092 the EDC (LCE2, IVL), as well as 30 other H3K9me3 peaks randomly chosen from

1093 group 1. (d-f) Hi-C contact maps at $40 \mathrm{~kb}$ resolution focused on the EDC locus in

1094 proliferating and RIS IMR90 cells (d), proliferating and replicative senescent WI38

1095 cells (e) as well as in differentiating keratinocytes (induced by calcium) (f); the arrows

1096 indicate the formation of loops during senescence (fibroblasts) and differentiation

1097 (keratinocytes) at this locus. (g-h) Hi-C contact maps on the NLRP3 locus at $40 \mathrm{~kb}$

1098 resolution in proliferating and RIS IMR90 cells (g), the arrow indicates the interaction

1099 changes around the gene body of NLRP3) and THP1 monocytes and PMA-induced

1100 macrophages (h). Hi-C maps in THP1 cells are aligned with H3K27ac ChIP-seq,

1101 ATAC-seq, and RNA-seq data.

1102

1103 
1104 Extended Data Figure 1. Transcriptional profile of genes in the EDC locus. (a)

1105 Image corrected, quantile normalized and log2 transformed microarray intensity

1106 values were downloaded from the indicated NCBI GEO entries. Control, proliferative;

1107 RIS, HRAS-G12V-induced senescent; Qui, quiescent; DDIS, DNA damage-induced

1108 senescent; acDDR, acute DNA damage response to etoposide (24 or 48 hours);

1109 E1A+RAS, transformed/senescence-bypassed. Genes for which there is no available

1110 transcript probe on the microarray platform used are greyed out. (b) Meta-analysis of

1111 senescence-associated transcriptomic changes of genes in the EDC locus using

1112 RNA-seq datasets, downloaded from indicated NCBI GEO, comparing between

1113 different cellular phenotypes. The datasets were processed using the same analysis

1114 pipeline. Values for the indicated genes are shown for each of the senescent

1115 samples and their corresponding growing controls. Values are Transcripts-Per-Million

1116 (TPM). Genes are ordered according to their position along Chr. 1. Genes for which

1117 there were no aligned reads in the specified RNA-seq runs are greyed out. (c)

1118 Immunoblot analysis of IMR90 cells stably expressing vector (-) or HRAS-G12V and

1119 control miR30 vector (-) or sh-p53 (+) for the proteins indicated. Arrow indicates

1120 specific bands. p21 is a p53-dependent cell cycle inhibitor that is upregulated during

1121 senescence. (d-e) IMR90 cells expressing ectopic mVenus-LCE2A and control

1122 vector were assessed for cumulative population doublings (d) and colony formation

1123 capacity $(e)(n=3)$. Error bars indicate mean $+/-$ SD. The inset shows representative

1124 immunoblots of indicated protein (d).

1125

1126 Extended Data Figure 2. Correlation between EDC decompaction and SAHF

1127 formation. (a) 3D confocal images of DNA-FISH for the EDC locus in the cells

1128 indicated (see Fig. 2a for two alternate color probe designs). The perinuclear

1129 localization of the EDC locus was quantified on visual inspections by counting FISH

1130 signal, which attached at least 1 of the 7 tiled signals to the nuclear periphery (white

1131 arrowhead) ( $n>100)$. (b) Representative images of DNA-FISH for the EDC and OR 
1132 loci using the probes indicated and quantification of signal length ( $n=100$ alleles

1133 from 3 biological replicates). Note, for the EDC, 5 out of 7 probes (Fig. 2a) were used.

1134 (c) FISH signal length of EDC on SAHF-negative or positive RIS cells ( $n \geq 100$ alleles

1135 from 3 biological replicates). (d) LCE2 immunofluorescence signal on SAHF-negative

1136 or positive RIS cells $(n=3)$. (e-h) Quantification of SAHF-positive cells in the

1137 indicated conditions (e, g) and comparative analysis of LCE2 expression by

1138 immunoblotting in the conditions indicated (f, h). ${ }^{*} P<0.05,{ }^{* *} P<0.01,{ }^{* *} P<0.001$

1139 one-way ANOVA with Tukey's multiple comparisons test $(n=3)$. Arrows indicate

1140 specific bands. (i) Quantification of FISH signal length in the conditions indicated for

1141 BJ and WI38 cells ( $n \geq 150$ alleles from 3 biological replicates). ${ }^{* * *} P<0.001$, Mann-

1142 Whitney-Wilcoxon test (bars are median with interquartile range) (b, c, i).

1143

1144 Extended Data Figure 3. H3K9me3 exhibits increases in accessibility and

1145 H3K27ac ChIP-seq signal in RIS. (a) ATAC-seq and H3K27ac THOR-normalized

1146 signal in proliferative and RIS cells centred on three classes of ATAC-seq peaks:

1147 proliferative-specific, RIS-specific, and common between the two conditions. (b)

1148 Number of ATAC-seq (top) and H3K27ac (bottom) peaks across the three groups of

1149 H3K9me3 peaks defined as permissive (group 2 and 3) and non-permissive (group

1150 1). The box plots correspond to the median, $25^{\text {th }}$ to $75^{\text {th }}$ percentiles, and the whiskers

1151 correspond to the $10^{\text {th }}$ to $90^{\text {th }}$ percentiles. (c) IGV tracks of genes of interest and

1152 position of SINE and Alu repeats (UCSC annotation) over their gene bodies. (d)

1153 Region surrounding the SERPINA1 gene characterised by H3K9me3 ChIP-seq

1154 signal, as well as protruding H3K27ac and ATAC-seq peaks.

1155

1156 Extended Data Figure 4. Tissue specificity of genes in H3K9me3 peaks. (a)

1157 Tissue specificity score of the genes within the three groups of H3K9me3 peaks

1158 against all other genes. The Tau score ranges from 0 - ubiquitous expression across

1159 tissues to 1 - tissue specific; the 8 genes of interest in group 3 are highlighted. 
1160 Significance testing was performed with two-sided Student's t-tests, ${ }^{* * *} P<0.001$,

1161 n.s., not significant. The box plots correspond to the median, $25^{\text {th }}$ to $75^{\text {th }}$ percentiles, 1162 and the whiskers correspond to the $10^{\text {th }}$ to $90^{\text {th }}$ percentiles. (b) DCC (left) and

1163 TMEM132B (right) genes represented by multiprobe DNA-FISH signals in the

1164 proliferative and RIS conditions. Probe sets are shown in Fig. 5b. Note, single color

1165 probe images are shown for TMEM132B.

1166

1167 Extended Data Figure 5. Three-dimensional interaction changes occurring

1168 during RIS. (a) $200 \mathrm{~kb}$ resolution $\mathrm{Hi}-\mathrm{C}$ maps in proliferative and RIS cells as well as

1169 significant interaction changes between the two conditions, where green arcs

1170 represent increases and blue arcs represent decreases. (b) Significant H3K9me3-

1171 H3K9me3 interaction changes (200 kb bins): log-fold changes (logFC) against

1172 distance between the interacting regions, dotted line corresponds to $10 \mathrm{Mb}$ distance.

1173 (c) Hi-C interaction profiles at $40 \mathrm{~kb}$ resolution in proliferating (Prolif) and RIS IMR90

1174 cells as well as differentiating keratinocytes at the EDC/OR loci. (d) Epigenetic profile

1175 of the NLRP3 locus in terms of H3K9me3, LaminB1 and H3K27me3 THOR-

1176 normalized ChIP-seq signal in proliferating and RIS cells. (e) Normalized gene

1177 expression (log-counts per million) of pro-inflammatory genes, EDC genes and

1178 senescence-representative genes in -/MSU and MSU/MSU synovial fibroblasts.

$1179 \quad{ }^{*}$ Adjusted $P<0.05,{ }^{* *} P<0.01,{ }^{* * *} P<0.001$.

1180

1181 Supplementary Table 1. List of genes within the three types of H3K9me3 peaks

1182 determined. 38 genes significantly upregulated in group 3 are highlighted.

1183

1184 


\section{Tomimatsu_Fig.1}

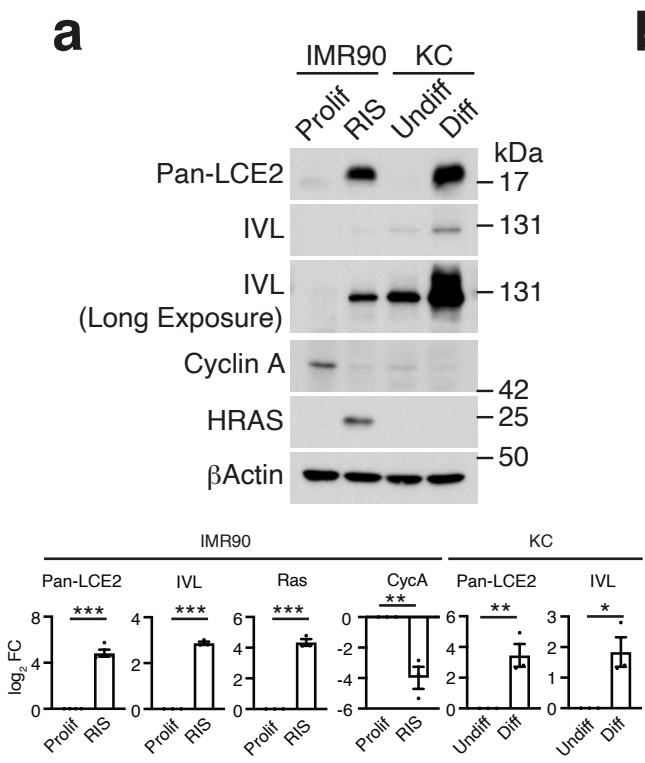

b

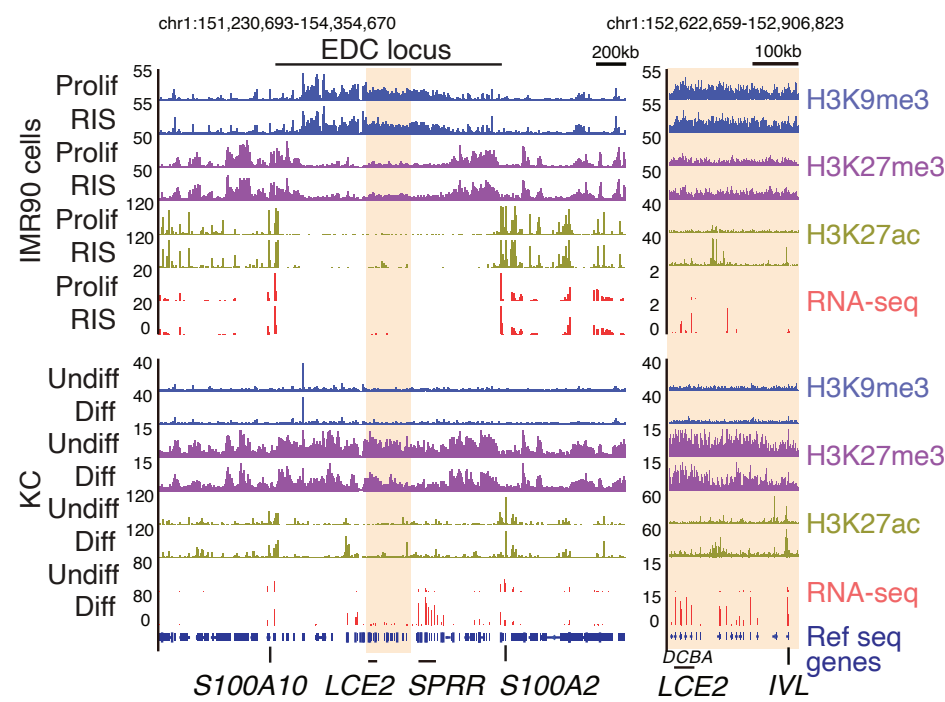

C

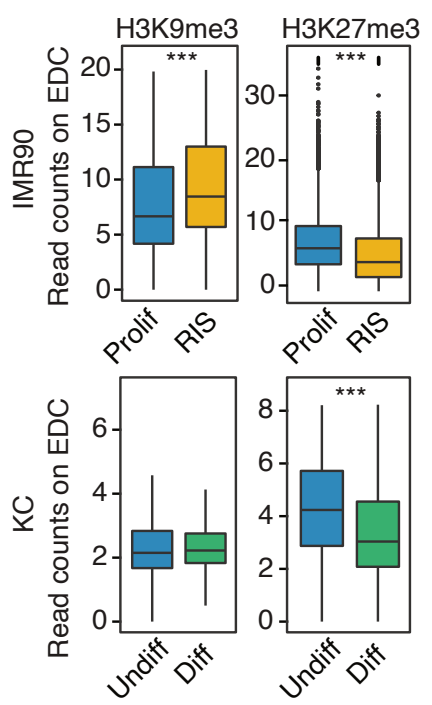

d
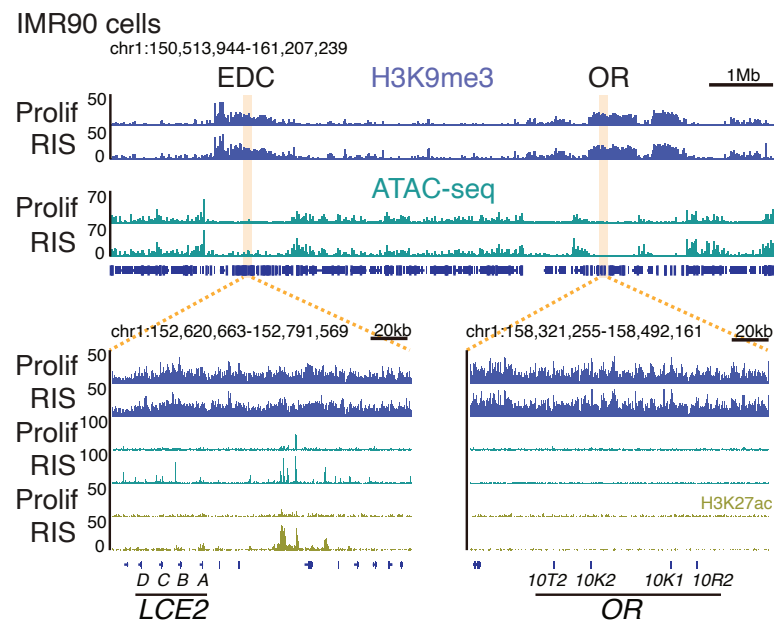

Figure 1. Aberrant expression of lineage-inappropriate LCE2 genes during senescence. (a) Comparative analysis of LCE2 expression by immunoblotting during fibroblast (IMR90) RIS (RAS-induced senescence) and keratinocyte (KC) differentiation (Diff). RIS was induced by retroviral expression of HRAS-G12V. Prolif, vector control proliferating cells. KC Diff was induced using PMA for 48h. Undiff, undifferentiated control. IVL, Involucrin, is an EDC-encoded KC Diff marker. Densitometry quantification was performed by ImageJ, normalized to BActin $(n \geq 3)$. ${ }^{*} P<0.05,{ }^{* *} P<0.01,{ }^{* * *} P<0.001$, unpaired t-test. (b) Representative genome browser images, centred around the EDC of the cells indicated. ER:HRASG12V expressing IMR90 cells were cultured with 4OHT (RIS) or without 4OHT (Prolif) for 6 days. ChIP-seq data were THOR-normalized. The region with the orange shading is magnified in the right-hand panel. (c) Averages of THORnormalized read counts for $\mathrm{H} 3 \mathrm{~K} 9 \mathrm{me} 3$ and $\mathrm{H} 3 \mathrm{~K} 27 \mathrm{me} 3$ signals on the EDC locus of the cells indicated. ${ }^{* \star *} P<0.001$, t-test and Shapiro-Wilkes normality test. (d) Representative genome browser images of ATAC-seq at the EDC and OR loci ( $\mathrm{n}$ $\geq 2$ ). The same ChIP-seq data are used as in $\mathbf{b}$. 
a
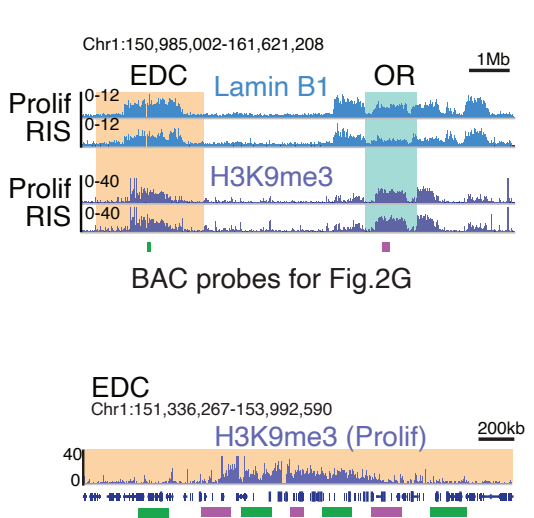

BAC probes for Fig.2B-D

b

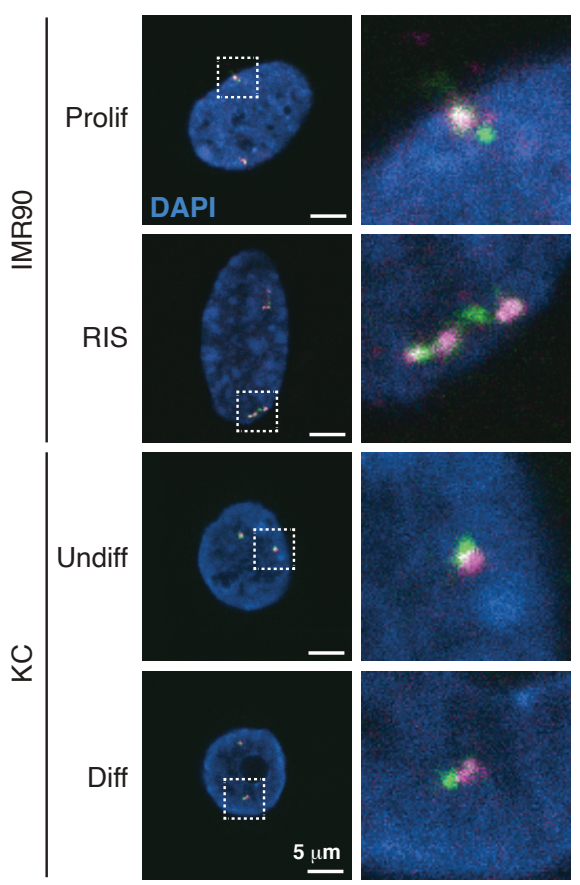

C

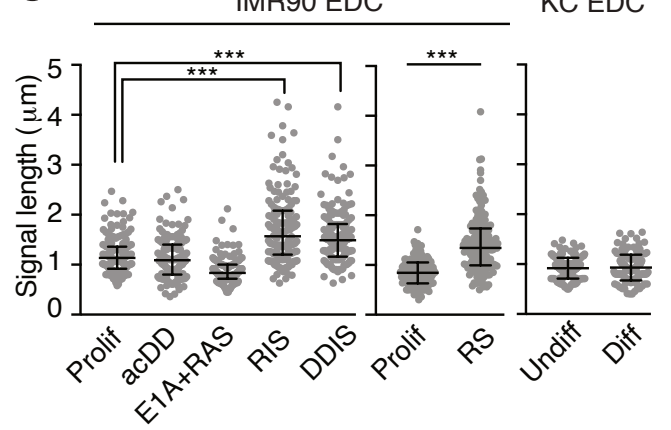

Tomimatsu_Fig.2

d IMR90 ER:HRASG12V

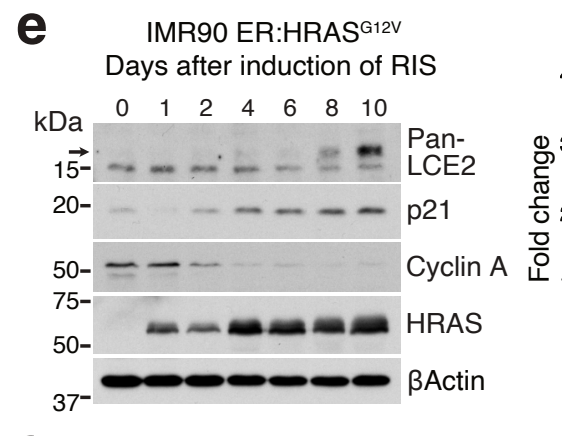

f

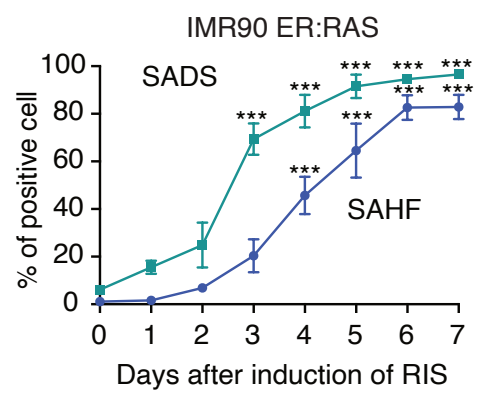

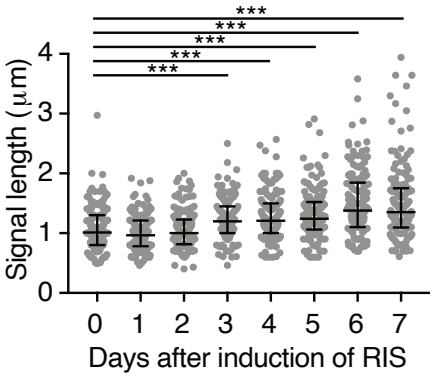

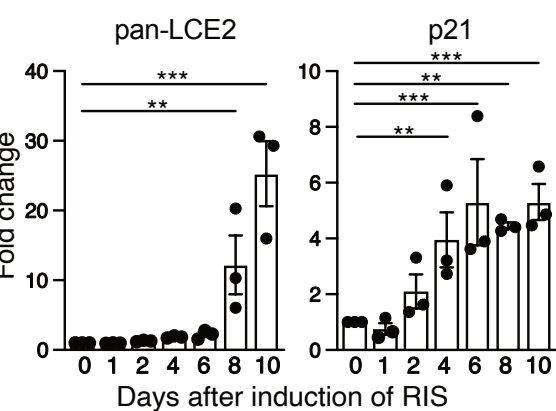

Days after induction of RIS
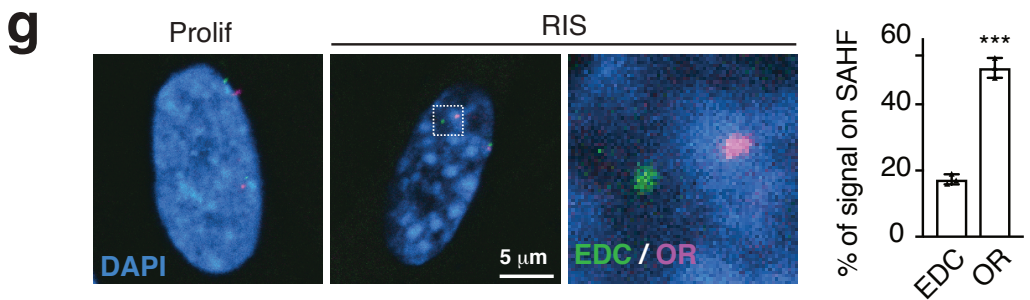

Figure 2. Decompaction of EDC locus in senescence IMR90 cells. (a) (a) Representative genome browser images of Lamin B1 ChIP-seq $(n=3)$ in proliferative and RIS IMR90 cells, with the location of BAC probes used in this study shown as green/purple blocks underneath. The same H3K9me3 ChIP-seq data as in Fig. 1 were used as the reference. (b) Confocal DNA-FISH images using multiple BAC probes tiled across the EDC locus as shown in a. (c) Quantification of the FISH signal length for the EDC locus; proliferating (Prolif), acute DNA damage (acDD), E1A and RAS expressing (E1A+RAS), RAS-induced senescence (RIS), DNA damage-induced senescence (DDIS), replicative senescence (RS) IMR90 cells; undifferentiated (Undiff) and differentiated (Diff) keratinocytes (KC). ${ }^{* *} P<0.001$, Mann-Whitney-Wilcoxon test $(n=150$ alleles from 3 biological replicates). Bars are median with inter-quartile range. (d) Time series analysis of EDC signal length following addition of $100 \mathrm{nM} 4 \mathrm{OHT}$ to ER:HRAS-G12V IMR90 cells. At least 150 alleles were counted from 3 biological replicates. ${ }^{* * *} P<0.001$ (Mann-Whitney-Wilcoxon test). Bars are median with interquartile range. (e) Representative immunoblotting of the time series experiments as in d. Arrow indicates specific bands. ${ }^{*} P<0.05,{ }^{* *} P<$ $0.01,{ }^{* *} P<0.001$ (one-way ANOVA with Fisher's LSD test, $\mathrm{n}=3$ ) (f) Quantification of SAHF- and SADS-positive nuclei of the time series experiments as in $\mathbf{d}$. Representative images of SAHF and SADS at d7 are shown. SADS are indicated by a-satellite targeted oligo hybridization (red). Values are means $+/-$ S.E.M. $(n=3)$. ${ }^{* *} P<0.001$ compared with d0, oneway ANOVA with Tukey's multiple comparisons test. (g) DNA-FISH using single BAC probes for EDC and OR loci as depicted in Fig. 2a (green and purple bars underneath top panel). Percentages of those loci colocalized with SAHF were measured. ${ }^{* * *} P<0.001$ unpaired t-test $(\mathrm{n}=3)$. 
a

b
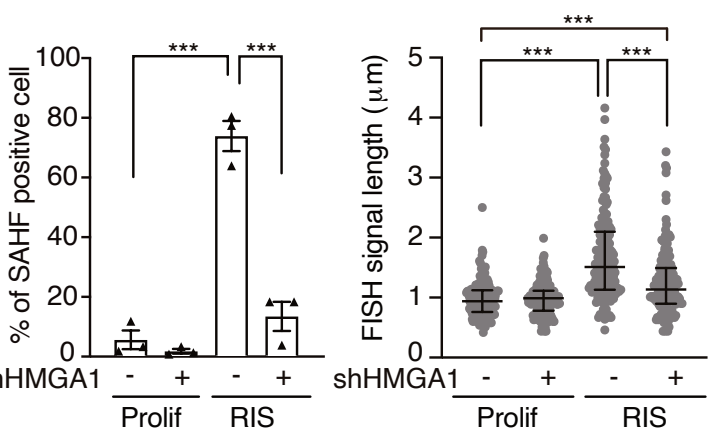

C

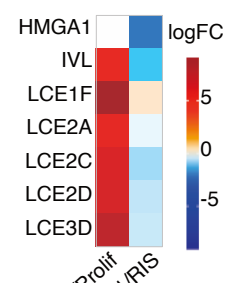

d

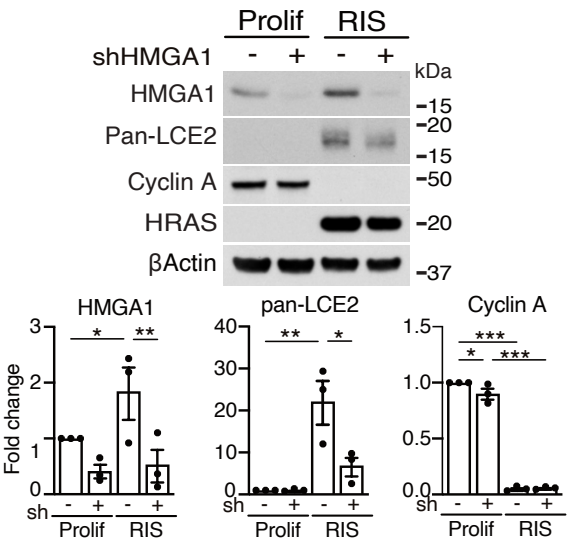

e

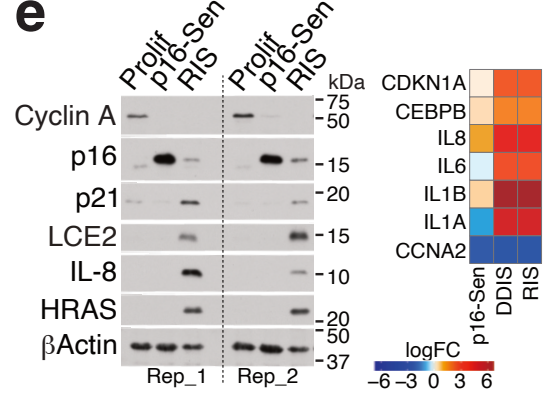

f

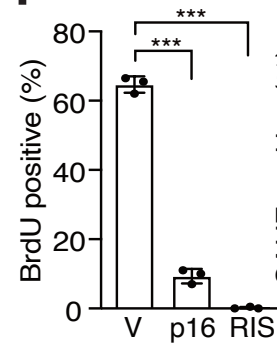

Tomimatsu_Fig. 3 $\mathrm{h}$

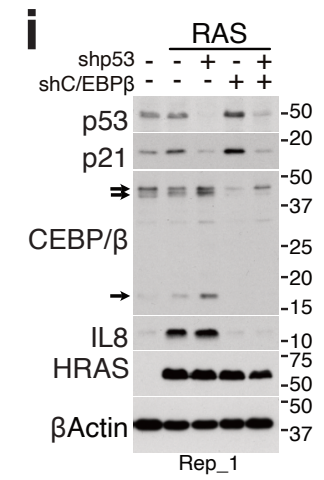

k
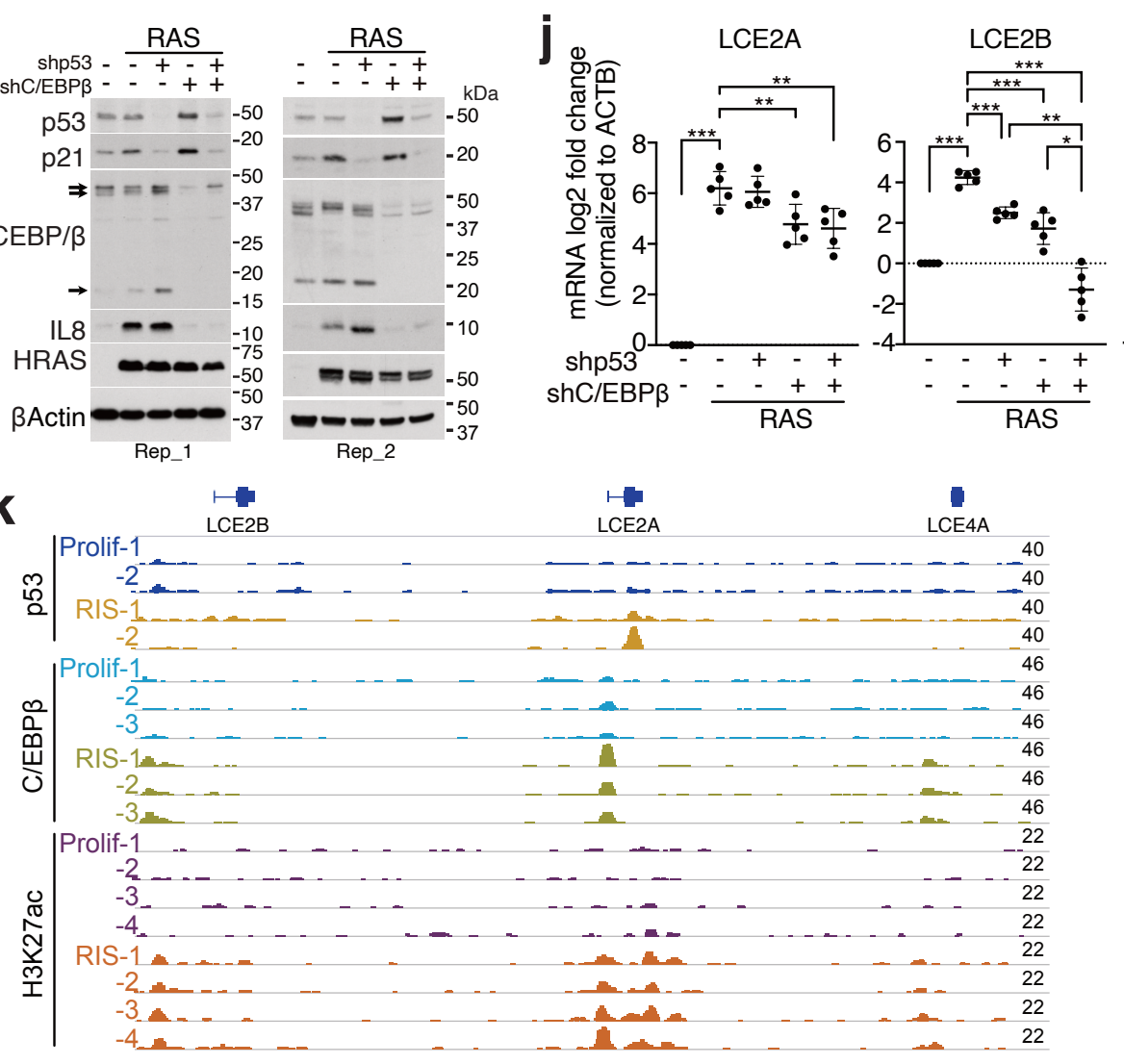

g

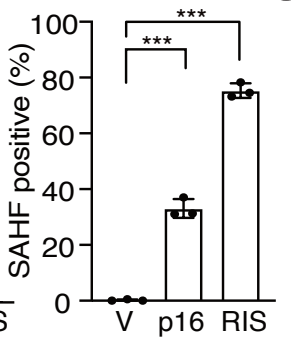

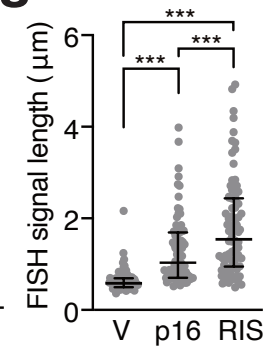

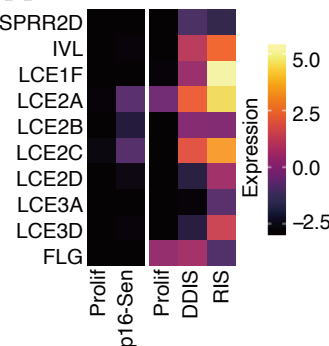

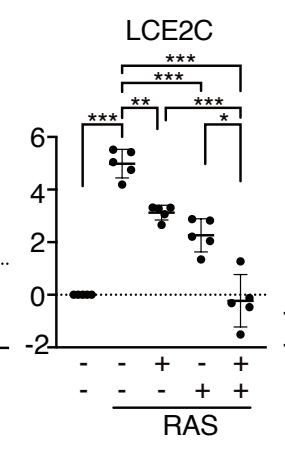

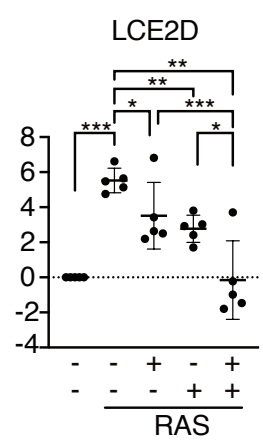


Figure 3. Decompaction of EDC locus and senescence effectors. (a) Quantification of SAHF-positive cells in RIS or proliferating (Vector) IMR90 cells with shHMGA1 (+) or control miR30 vector (-). (b) Quantification of the FISH signal length for the EDC locus in the indicated cells. (c) RNA-seq analysis in the indicated comparison. (d) Immunoblotting for the indicated proteins. ${ }^{\star} P<0.05$, ${ }^{\star \star} P<0.01$, ${ }^{\star \star \star} P<0.001$, (one-way ANOVA with Fisher's LSD test, $\mathrm{n}=3$ ). (e) Left, immunoblotting for the indicated proteins in IMR90 cells expressing control retroviral vector (Prolif), p16 (p16-Sen), and HRAS-G12V (RIS). Two representative replicates are shown. Right, corresponding RNA-seq analysis, showing log-fold change (logFC) in the indicated conditions compared to corresponding proliferative controls. p16-Sen $(n=3)$, DDIS $(n=$ 8), RIS ( $\mathrm{n}=8)$. (f) Quantification of BrdU incorporation and SAHF-positive cells. Values are means $+/-\operatorname{SD}(n=3)$. ${ }^{* \star *} P<$ 0.001, (one-way ANOVA with Fisher's LSD test). (g) Quantification of the FISH signal length for the EDC locus in the indicated cells. ${ }^{\star \star \star} \mathrm{P}<0.001$, (ordinary one-way ANOVA with Fisher's LSD test, $n \geq 80$ allele from 3 biological replicates). Bars are median with inter-quartile range. (h) RNA-seq analysis - normalized expression heatmap (log-counts per million) of EDC genes in p16-induced senescence (p16-Sen), DNA damage-induced senescence (DDIS) and RAS-induced senescence (RIS) with corresponding proliferative controls. (I - j) Two representative immunoblots $(n \geq 5)$ Arrows indicate the three specific isoforms. (i) and qPCR analysis (j) in indicated cells. ER:RAS IMR90 cells expressing shp53 and shC/ EBP $\beta$ with corresponding control vectors were treated with 4OHT (RAS) for 6 days. Values are means $+/-\mathrm{SD}(\mathrm{n}=5)$. ${ }^{\star} P$ $<0.05,{ }^{\star \star} P<0.01,{ }^{\star \star \star} P<0.001$, (one-way ANOVA with Fisher's LSD test). (k) IGV tracks of THOR-normalized ChIP-seq signal of p53, C/EBP $\beta$ and H3K27ac in Proliferating (Prolif) and RIS samples. (I) Quantification of the FISH signal length for the EDC locus in the indicated cells. ${ }^{\star * *} P<0.001$, Mann-Whitney-Wilcoxon test $(n \geq 150$ alleles from 3 biological replicates). n.s., not significant. 

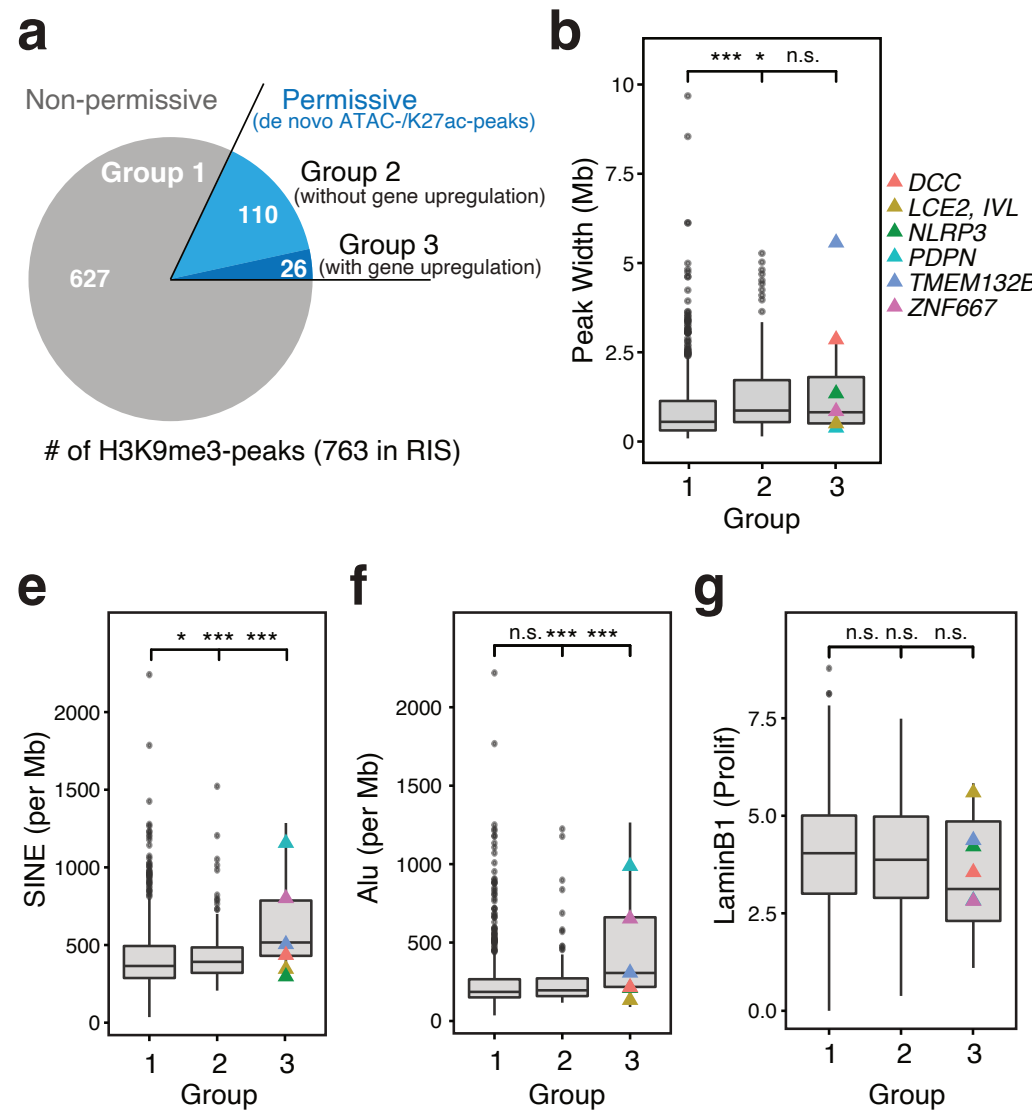

f

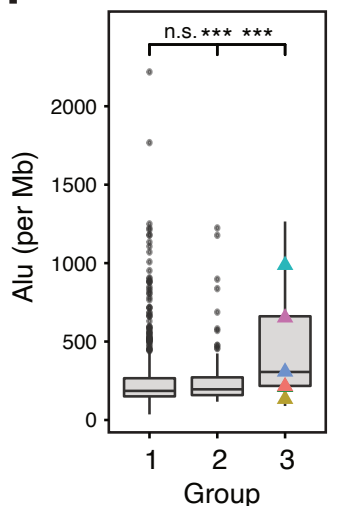

g

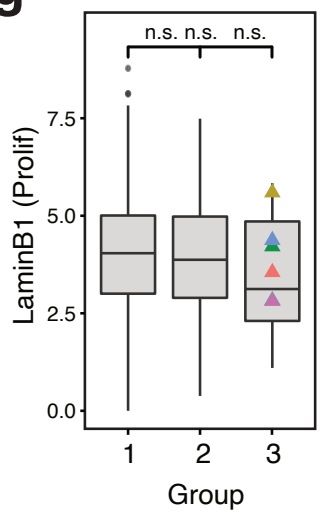

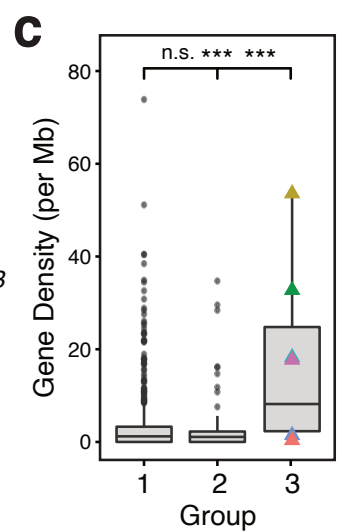

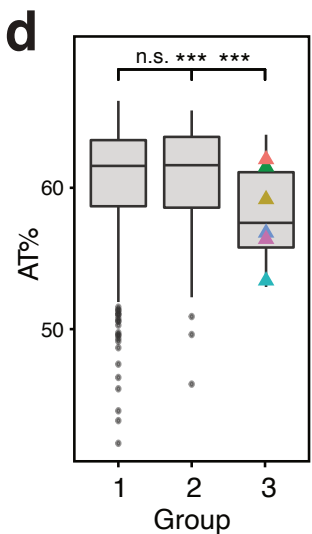

h

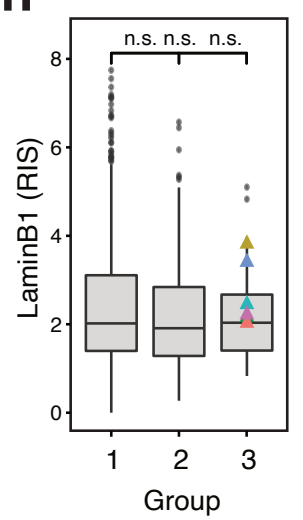

i

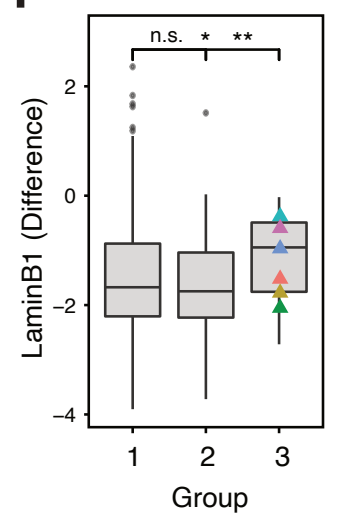

Figure 4. H3K9me3 peaks characterisation. (a) Classification of H3K9me3 peaks into non-permissive (group 1) and permissive peaks (with de novo ATAC-seq and H3K27ac peaks) with (group 3) and without up-regulated genes (group 2). (bi) Distribution of peak widths (Mb) (b), gene density (per Mb) (c), AT\% (d), SINE frequency (e), Alu repeats frequency (f), Lamin B1 THOR-normalized ChIP-seq signal in proliferating cells (g), Lamin B1 THOR-normalized ChIP-seq signal in RIS cells (h), differential LaminB1 signal between RIS and proliferative cells (i). In group 3, H3K9me3-peaks associated with the genes of interest are highlighted $(\mathbf{b}-\mathbf{i})$. All box plots correspond to the median, $25^{\text {th }}$ to $75^{\text {th }}$ percentiles, and the whiskers correspond to the $10^{\text {th }}$ to $90^{\text {th }}$ percentiles. Each pairwise comparison between groups was effectuated with a two-sided Student's t-test, ${ }^{* * *} P<0.001,{ }^{* *} P<0.01,{ }^{*} P<0.05$. n.s., not significant. 


\section{Tomimatsu_Fig. 5}

a

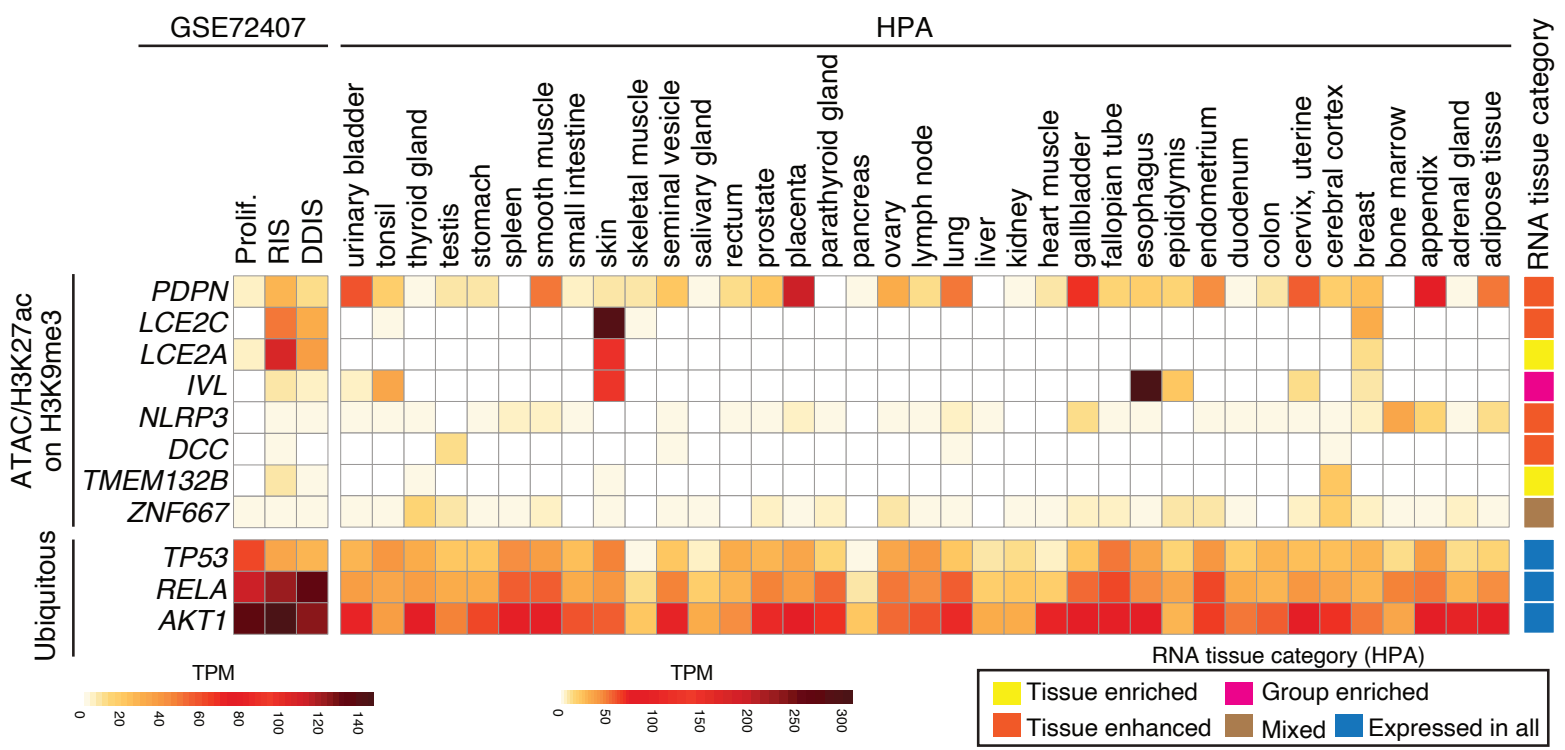

b
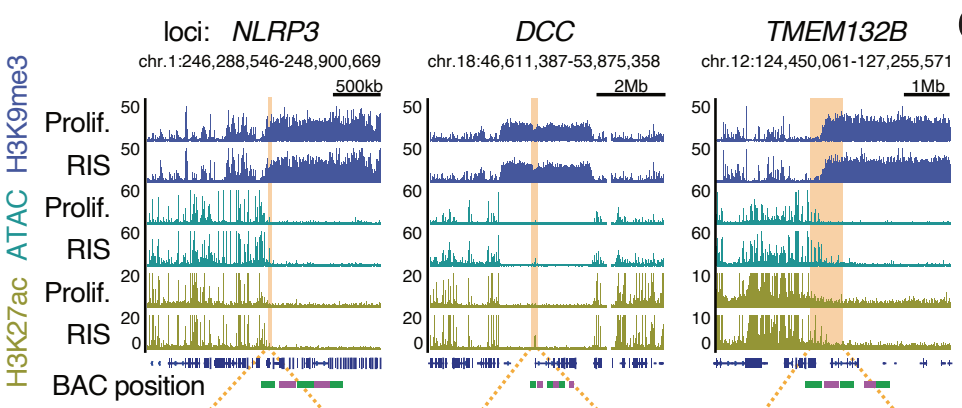

C
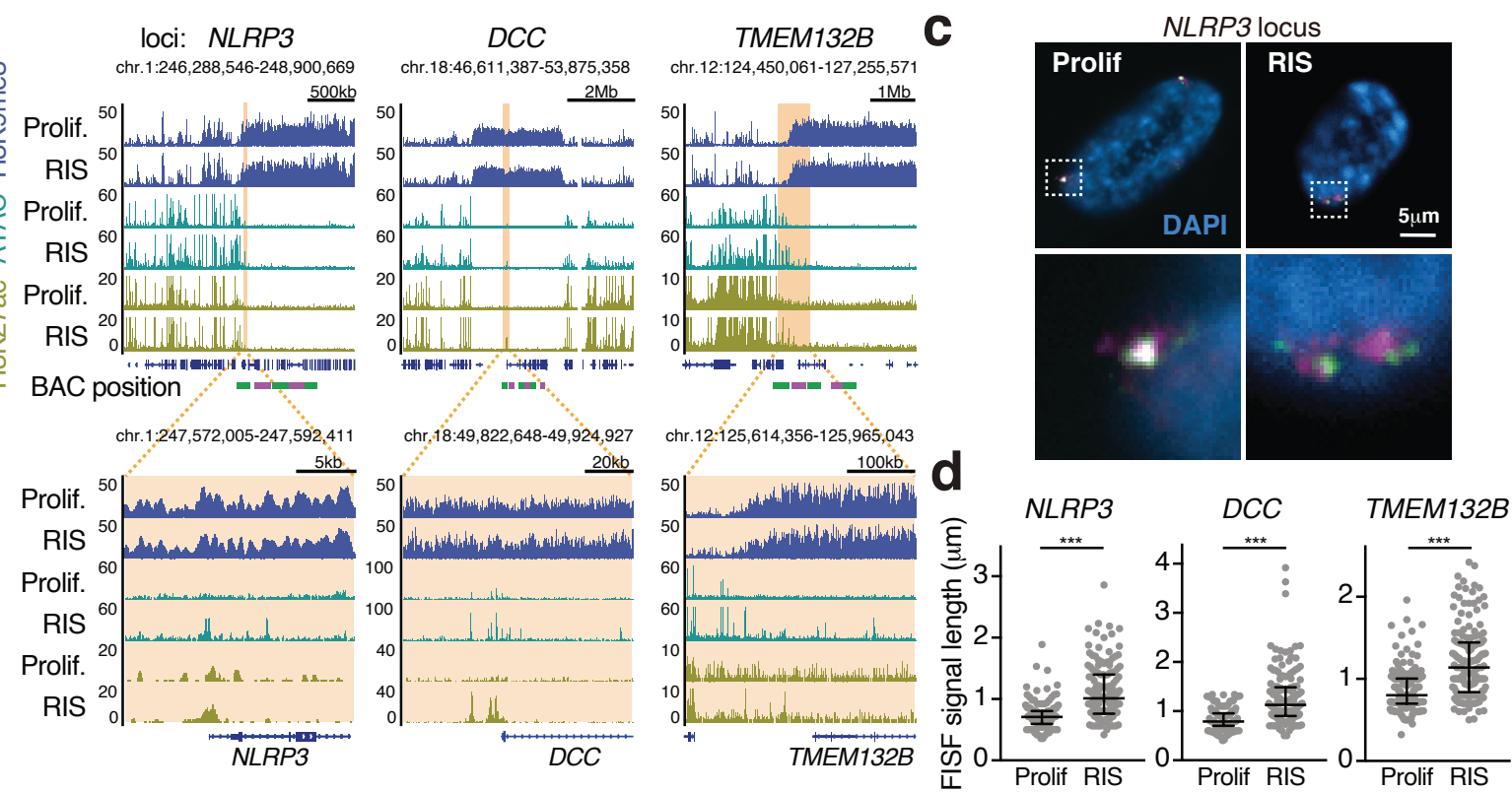

Figure 5. Aberrant expression of lineage-specific genes and chromatin decompaction during senescence. (a) Tissue-dependent expression pattern of RIS upregulated genes that have RIS-unique H3K27ac/ATAC-peaks (at TSS +/- 250 bp) within H3K9me3 domains. RNA-seq datasets from IMR90 for RIS and DDIS (GSE-72407) and Human Protein Atlas (HPA) RNA-seq for normal tissues (E-MTAB-2836) were used. TPM, Transcripts-Per-Million. (b-d) Chromatin states of three additional examples of upregulated genes from H3K9me3 regions during RIS. Representative genome browser images (b). Representative multiprobe DNA-FISH images for the NLRP3 locus (c). Either single or two alternate colours were used in the probe set shown in $\mathbf{b}$ (green/purple blocks, underneath main panel). Measurement of FISH signals using the multiple BAC probes represented in (d). ${ }^{* * *} P<0.001$, Mann-Whitney-Wilcoxon test $(\mathrm{n} \geq 150$ alleles from 3 biological replicates). Bars, median with interquartile range. 


\section{Tomimatsu_Fig. 6}

a
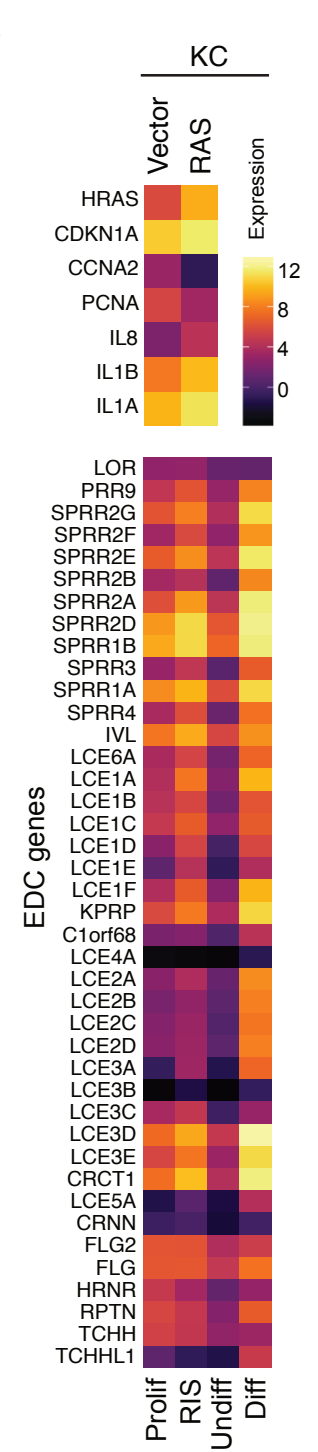

b

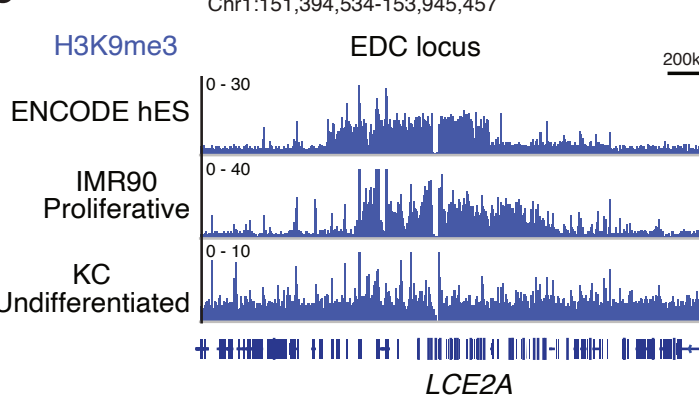

Chr18:47,226,915-53,423,201

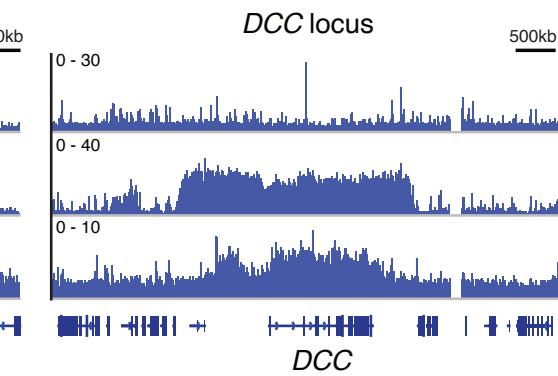

EDC

C

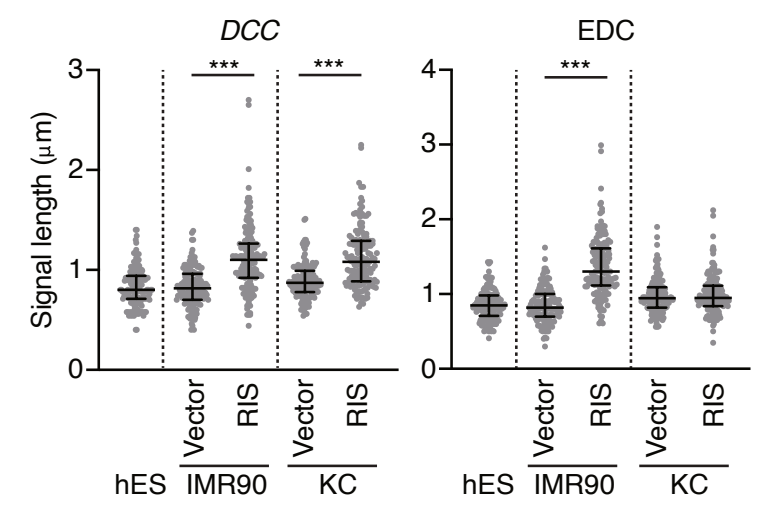

KC

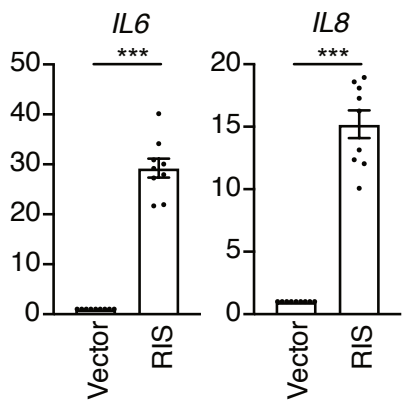

Figure 6. Distinct mode of expression of lineage-appropriate and lineage-inappropriate genes during RIS. (a) Heatmap of normalized expressionvalues (log-counts per million) of the EDC genes from RNA-seq samples of proliferating and RASinduced $(n=6)$ keratinocytes (KCs), as well as undifferentiated $(n=8)$ and differentiated $(n=9)$ KCs. (b) Genome browser images of H3K9me3 ChIP-seq of the EDC and DCC loci from the cells indicated. The human ES (hES) cell data are from ENCODE. Some of the IMR90 and human primary keratinocyte $(K C)$ images were replotted for comparisons using the same datasets as in Fig. $1 \mathrm{~b}$ (EDC) and Fig. $5 \mathrm{~b}(D C C)$. (c) DNA-FISH analysis of the $D C C$ and EDC loci in the cells indicated. ${ }^{* * *} P<$ 0.001 , Mann-Whitney-Wilcoxon test ( $\mathrm{n} \geq 150$ alleles from 3 biological replicates). Bars are median with inter-quartile range. (d) qRT-PCR for the indicated mRNA in vector control and RIS KCs. Error bars indicate mean $+/$ - S.E.M. $(n=9) .{ }^{* \star} P<0.001,{ }^{*} P$ $<0.05$ paired t-test. 


\section{Tomimatsu_Fig. 7}
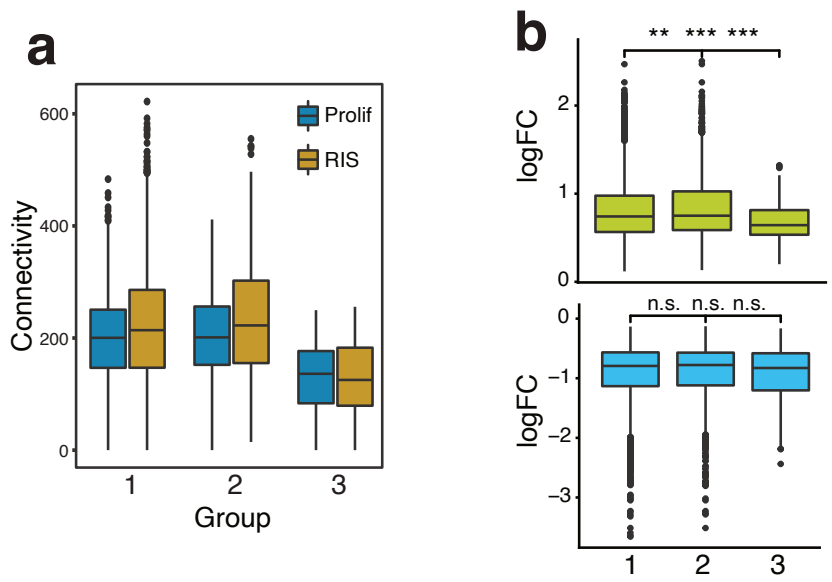

C
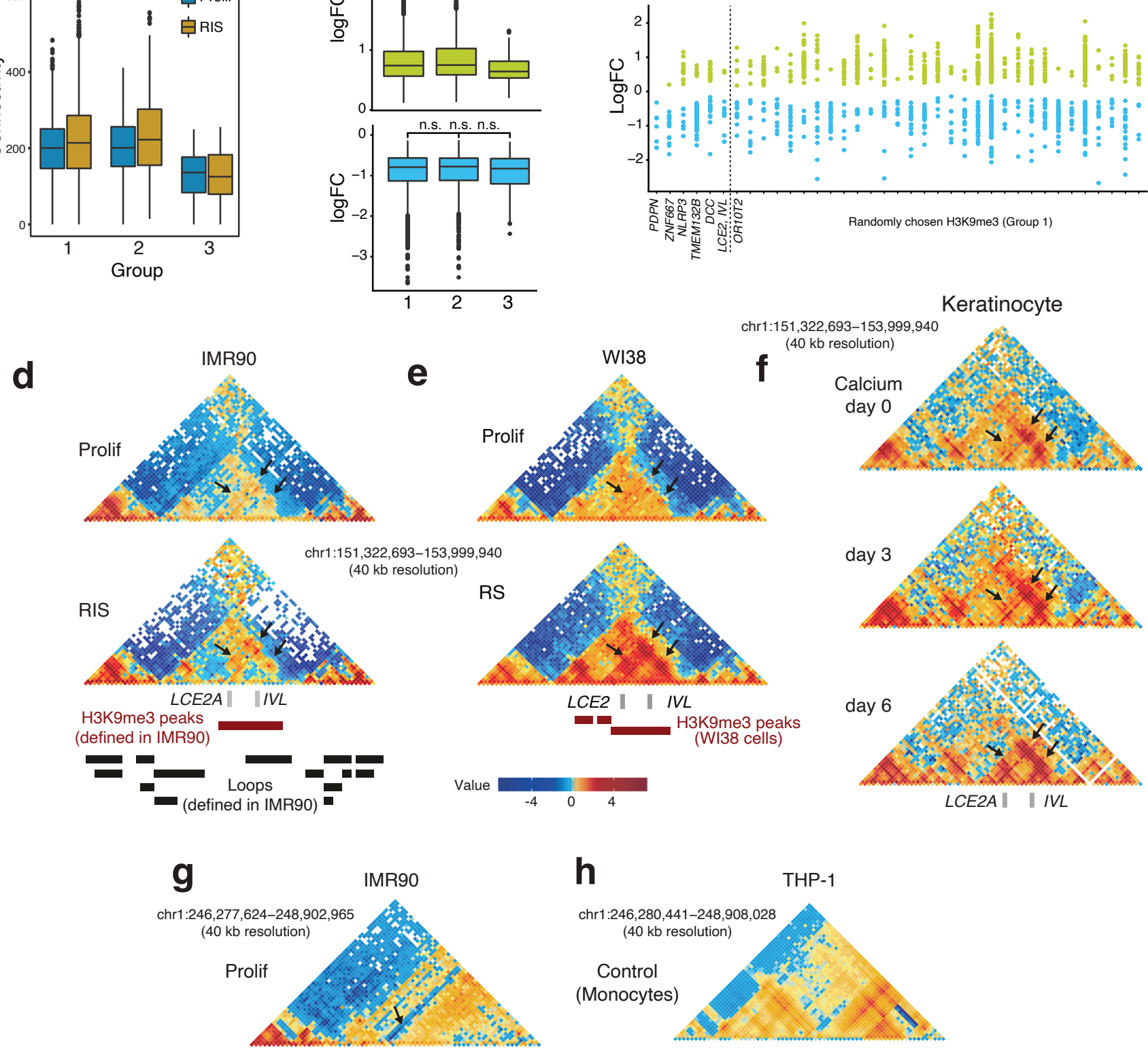

h
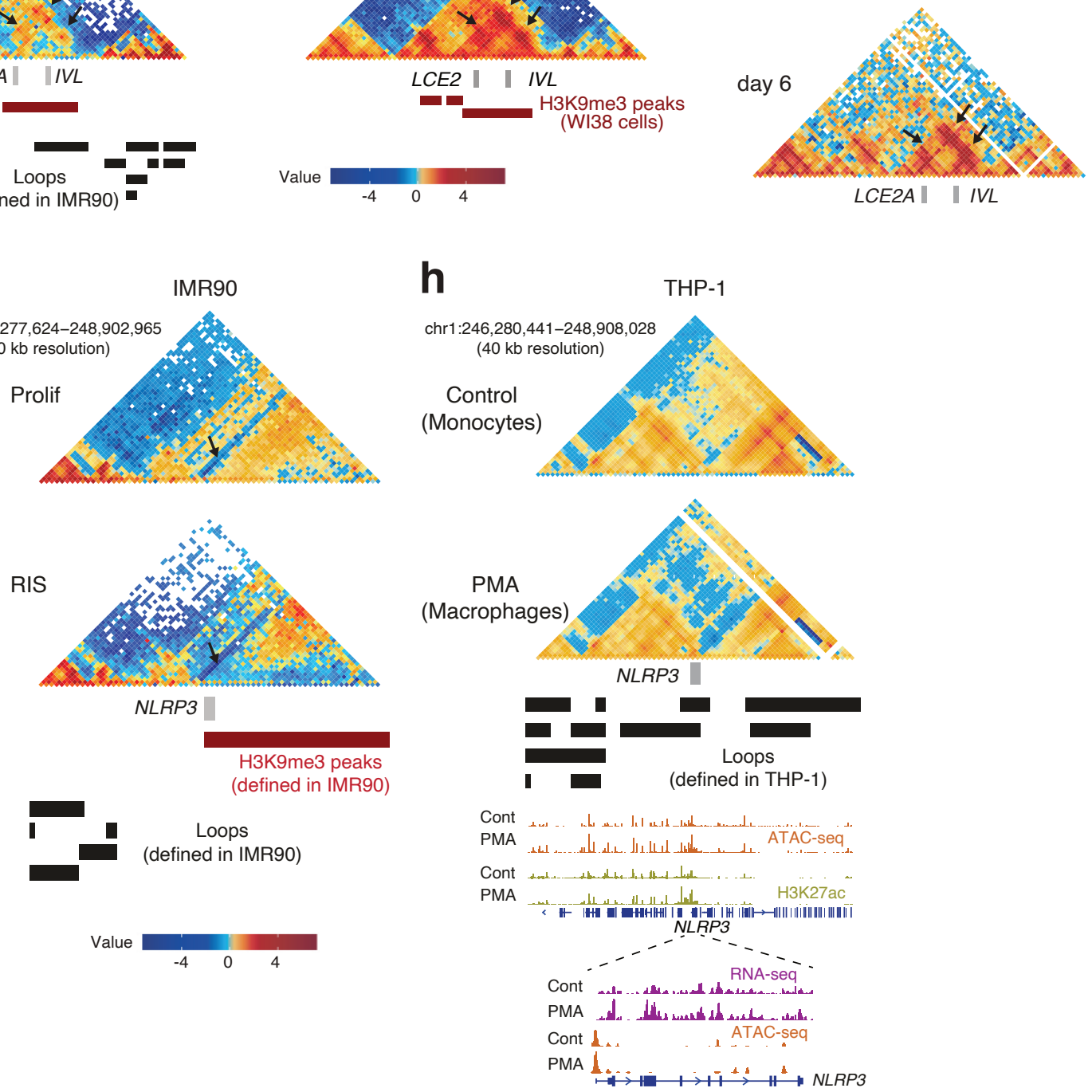
Figure 7. Differential 3D behaviour of permissive H3K9me3 peaks. (a) Total H3K9me3-H3K9me3 interactions (connectivity) of the individual $200 \mathrm{~kb}$ bins (sum of the interactions with all other $200 \mathrm{~kb}$ bins within $\mathrm{H} 3 \mathrm{~K} 9 \mathrm{me} 3$ peaks) within each group of $\mathrm{H} 3 \mathrm{~K} 9 \mathrm{me} 3$ peaks (the same three groups as in Fig. 4). (b) Differential H3K9me3-H3K9me3 interaction between proliferative and RIS conditions in each group. Log-fold changes (logFC) of increased (top, green) and decreased interactions (bottom, blue) in each peak with all other H3K9me3 peaks are shown. Each pairwise comparison is performed using two-sided Student's t-test with ${ }^{\star \star *} P<0.001$, ${ }^{\star *} P<0.01$. n.s., not significant. Boxplots in (a) and (b) represent the median, 25th to 75th percentiles, whiskers: 10th to 90th percentiles. (c) Significant LogFC (green - positive, blue - negative) of all the $\mathrm{Hi}-\mathrm{C}$ interactions involving each of the $\mathrm{H} 3 \mathrm{~K} 9 \mathrm{me} 3$ peaks of interest within group 3 compared to the OR region (OR10T2) neighbouring the EDC (LCE2, IVL), as well as 30 other $\mathrm{H} 3 \mathrm{~K} 9 \mathrm{me} 3$ peaks randomly chosen from group 1. (d-f) Hi-C contact maps at $40 \mathrm{~kb}$ resolution focused on the EDC locus in proliferating and RIS IMR90 cells (d), proliferating and replicative senescent WI38 cells (e) as well as in differentiating keratinocytes (induced by calcium) (f); the arrows indicate the formation of loops during senescence (fibroblasts) and differentiation (keratinocytes) at this locus. (g-h) Hi-C contact maps on the NLRP3 locus at $40 \mathrm{~kb}$ resolution in proliferating and RIS IMR90 cells $(\mathrm{g})$, the arrow indicates the interaction changes around the gene body of NLRP3) and THP1 monocytes and PMA-induced macrophages (h). Hi-C maps in THP1 cells are aligned with H3K27ac ChIP-seq, ATAC-seq, and RNA-seq data. 


\section{Tomimatsu_Extended Data Fig. 1}

a

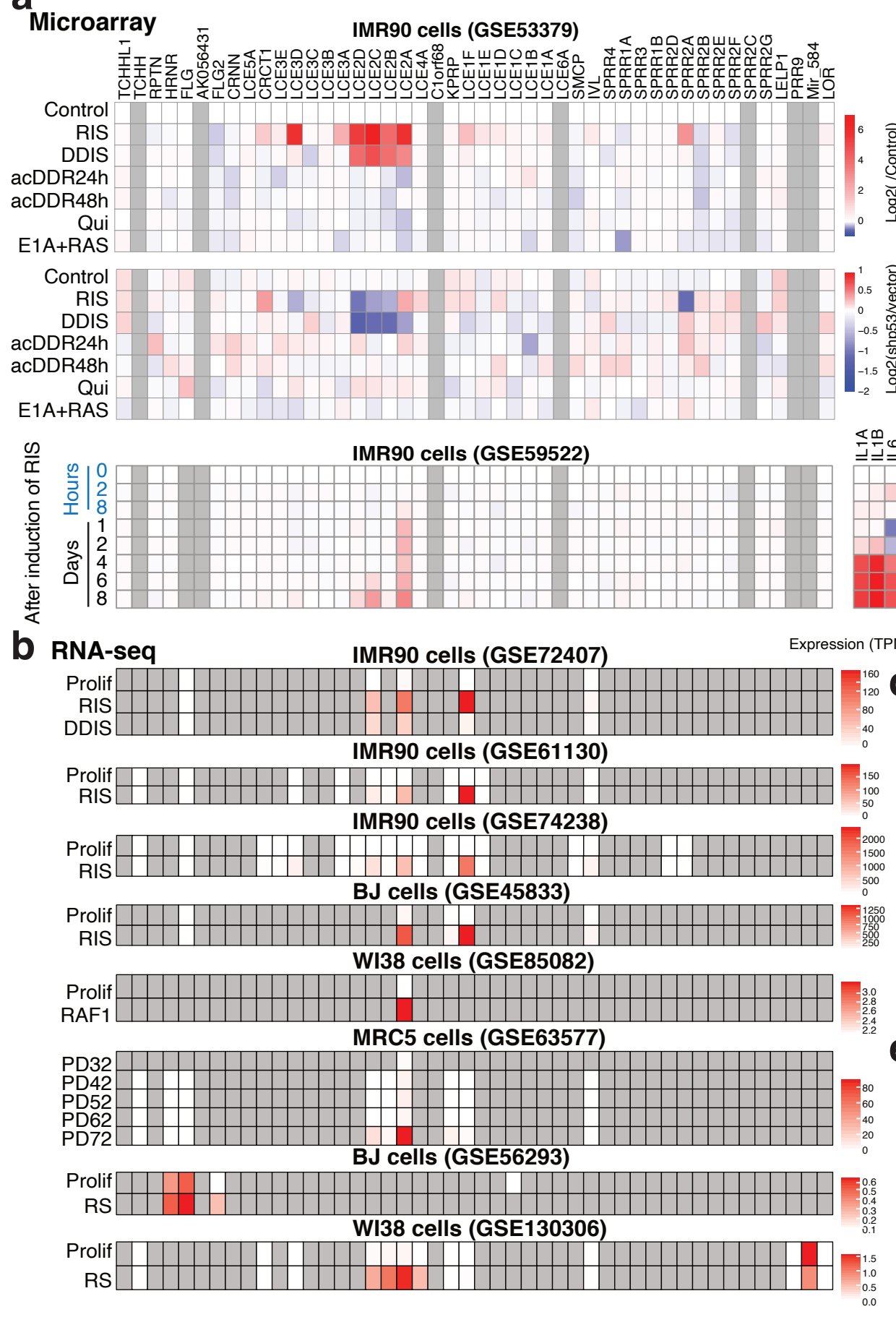

C

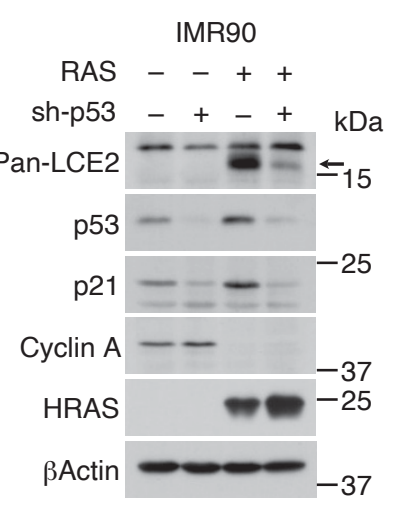

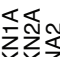
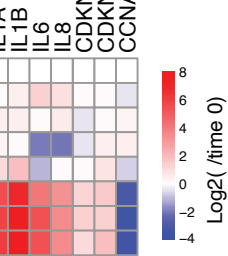

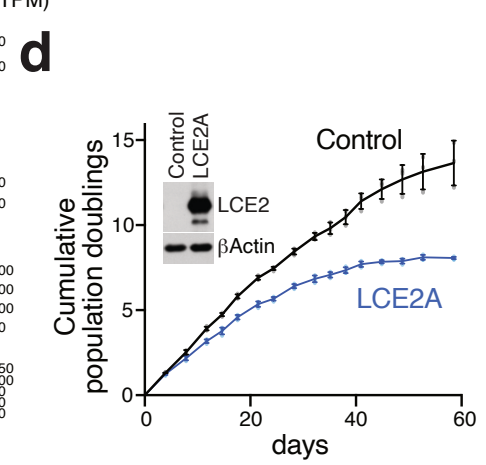

e

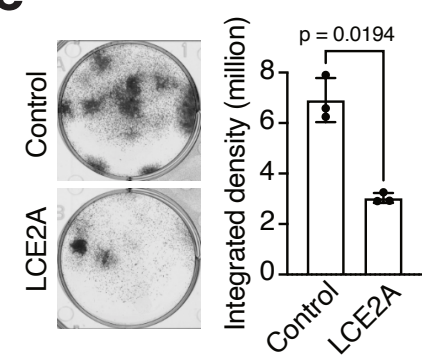

Extended Data Figure 1. Transcriptional profile of genes in the EDC locus. (a) Image corrected, quantile normalized and log2 transformed microarray intensity values were downloaded from the indicated NCBI GEO entries. Control, proliferative; RIS, HRAS-G12V-induced senescent; Qui, quiescent; DDIS, DNA damage-induced senescent; acDDR, acute DNA damage response to etoposide ( 24 or 48 hours); E1A+RAS, transformed/senescence-bypassed. Genes for which there is no available transcript probe on the microarray platform used are greyed out. (b) Meta-analysis of senescenceassociated transcriptomic changes of genes in the EDC locus using RNA-seq datasets, downloaded from indicated NCBI GEO, comparing between different cellular phenotypes. The datasets were processed using the same analysis pipeline. Values for the indicated genes are shown for each of the senescent samples and their corresponding growing controls. Values are Transcripts-Per-Million (TPM). Genes are ordered according to their position along Chr. 1. Genes for which there were no aligned reads in the specified RNA-seq runs are greyed out. (c) Immunoblot analysis of IMR90 cells stably expressing vector (-) or HRAS-G12V and control miR30 vector (-) or sh-p53 (+) for the proteins indicated. Arrow indicates specific bands. p21 is a p53-dependent cell cycle inhibitor that is upregulated during senescence. (d-e) IMR90 cells expressing ectopic mVenus-LCE2A and control vector were assessed for cumulative population doublings (d) and colony formation capacity $(e)(n=3)$. Error bars indicate mean $+/-$ SD. The inset shows representative immunoblots of indicated protein (d). 


\section{Tomimatsu_Extended Data Fig. 2}

a

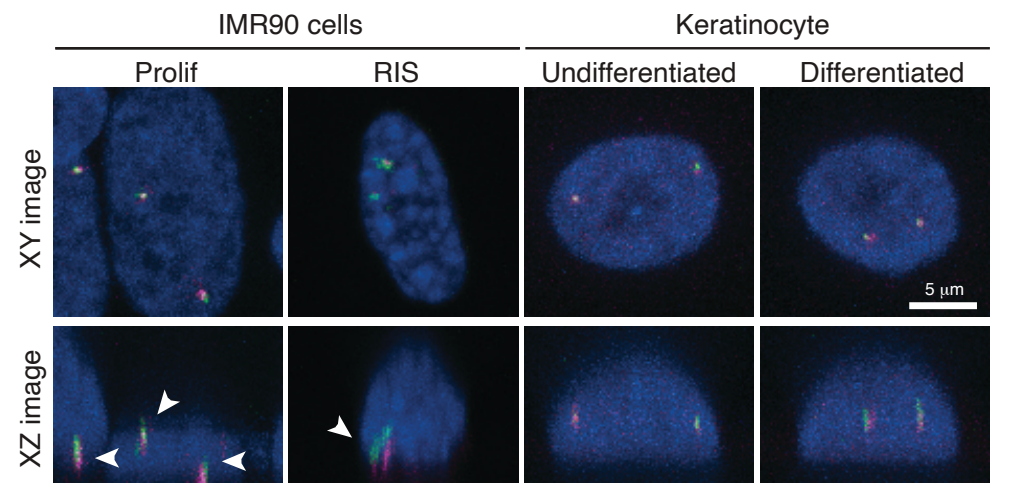

b

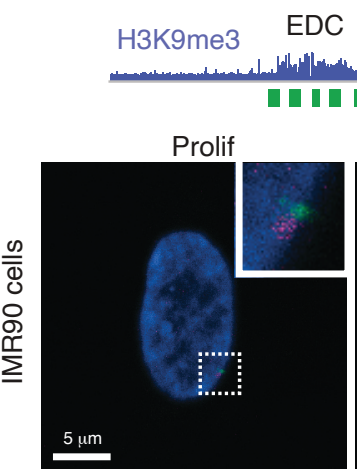

DC
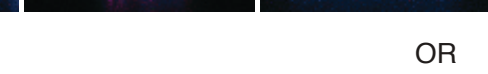

$\mathrm{OR} \quad 1 \mathrm{Mb}$
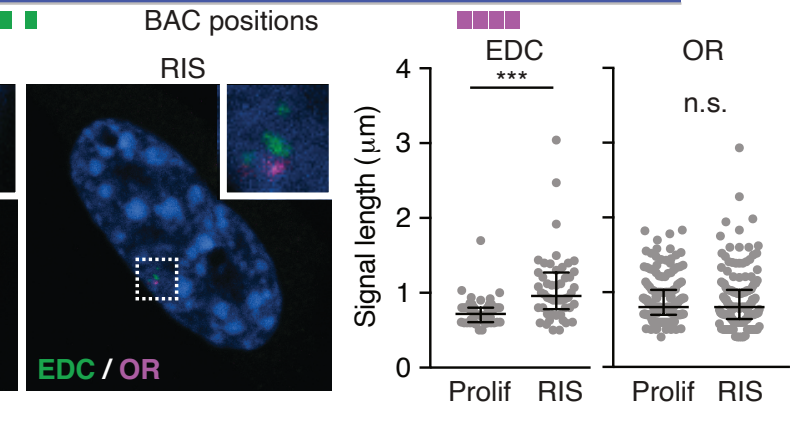

C

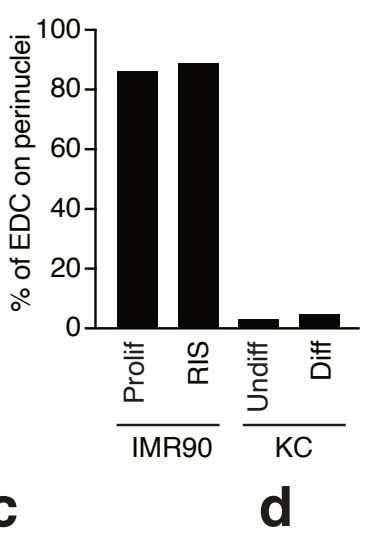

e
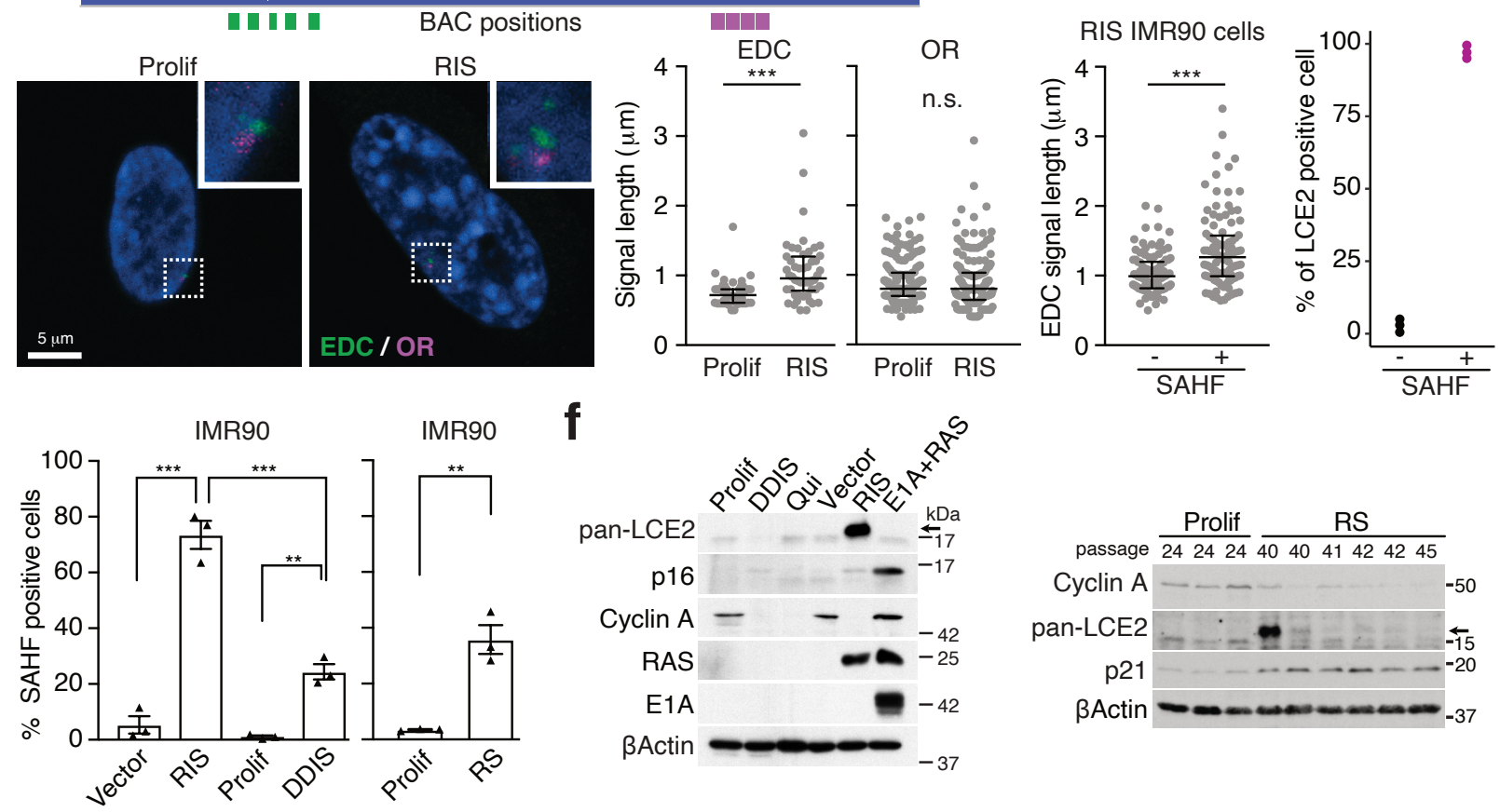

g

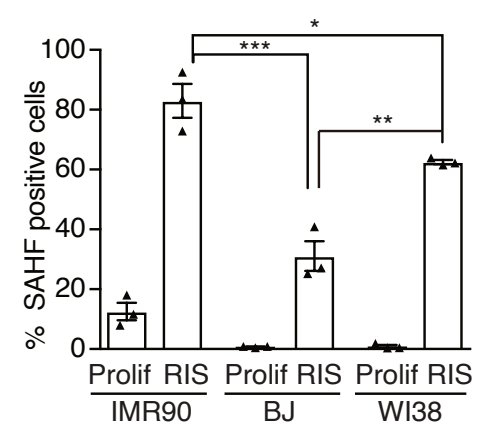

h

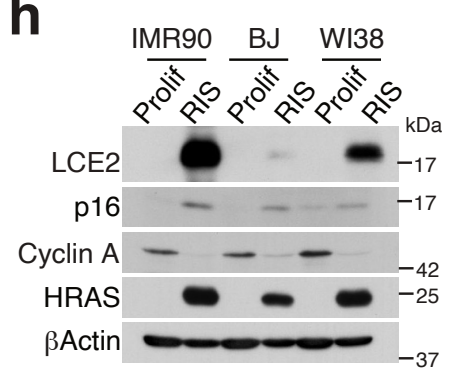

i
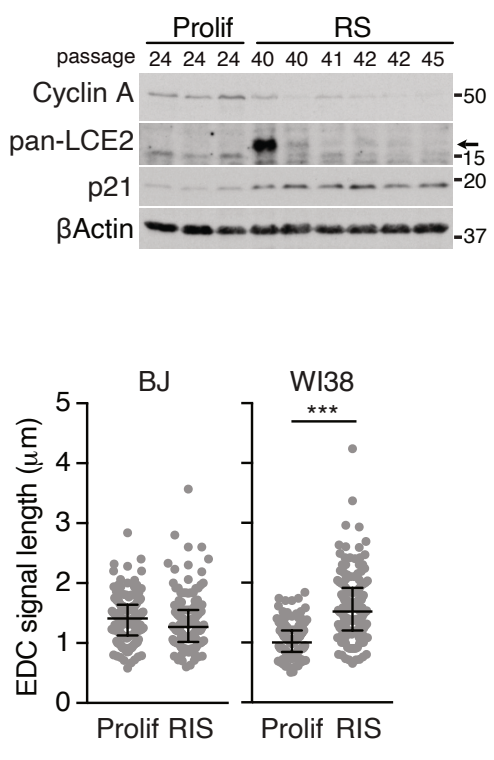

Extended Data Figure 2. Correlation between EDC decompaction and SAHF formation. (a) 3D confocal images of DNA-FISH for the EDC locus in the cells indicated (see Fig. 2a for two alternate colour probe designs). The perinuclear localization of the EDC locus was quantified on visual inspections by counting FISH signal, which attached at least 1 of the 7 tiled signals to the nuclear periphery (white arrowhead) $(n>100)$. (b) Representative images of DNA-FISH for the EDC and OR loci using the probes indicated and quantification of signal length ( $n=100$ alleles from 3 biological replicates). Note, for the EDC, 5 out of 7 probes (Fig. 2a) were used. (c) FISH signal length of EDC on SAHF-negative or positive RIS cells ( $n \geq 100$ alleles from 3 biological replicates). (d) LCE2 immunofluorescence signal on SAHF-negative or positive RIS cells $(n=3)$. $(e-h)$ Quantification of SAHF-positive cells in the indicated conditions $(e, g)$ and comparative analysis of LCE2 expression by immunoblotting in the conditions indicated (f, h). ${ }^{*} P<0.05,{ }^{\star \star} P<0.01,{ }^{* \star *} P<0.001$ one-way ANOVA with Tukey's multiple comparisons test $(n=3)$. Arrows indicate specific bands. (i) Quantification of FISH signal length in the conditions indicated for BJ and WI38 cells ( $\mathrm{n} \geq 150$ alleles from 3 biological replicates). ${ }^{* *} P<0.001$, Mann-Whitney-Wilcoxon test (bars are median with interquartile range) (b, c, i). 


\section{Tomimatsu_Extended Data Fig. 3}

a

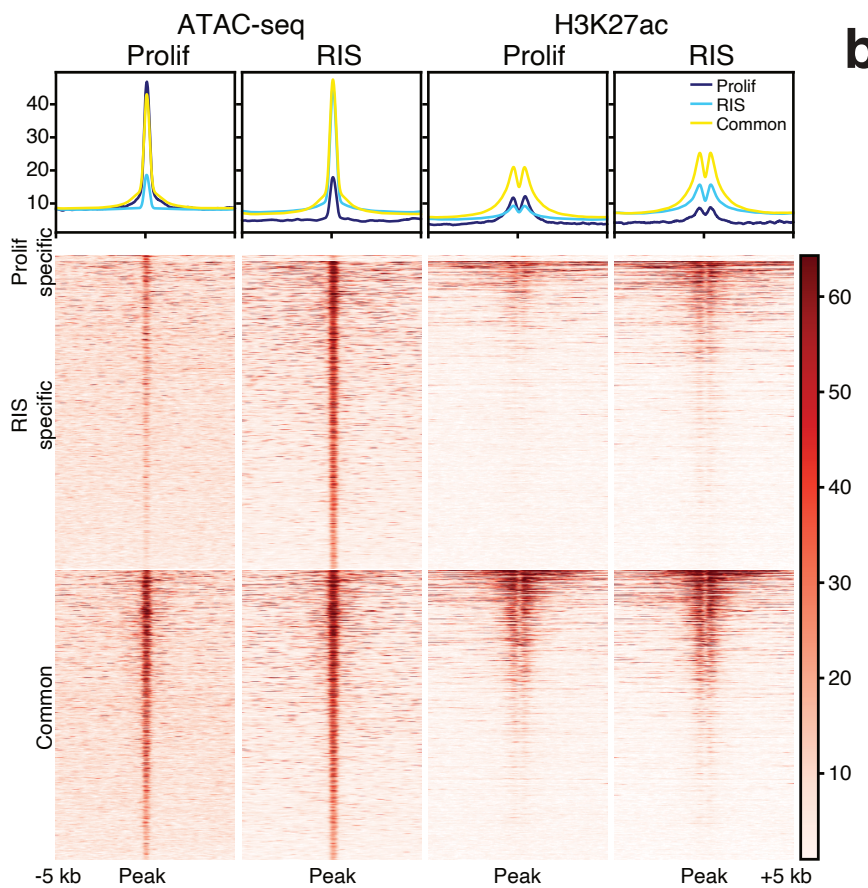

C

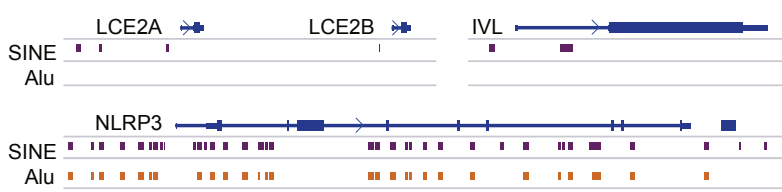

Alu || I| | | || || |" | | |"

III I II I ।
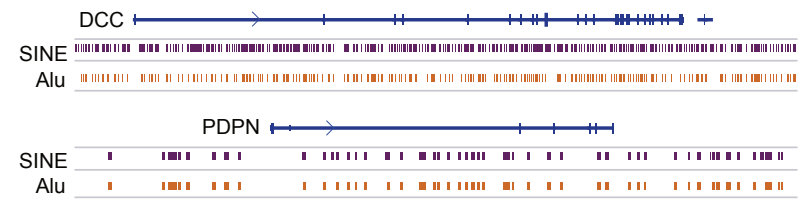

TMEM132B

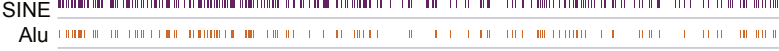

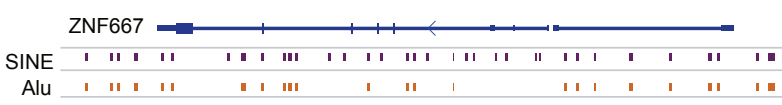

b
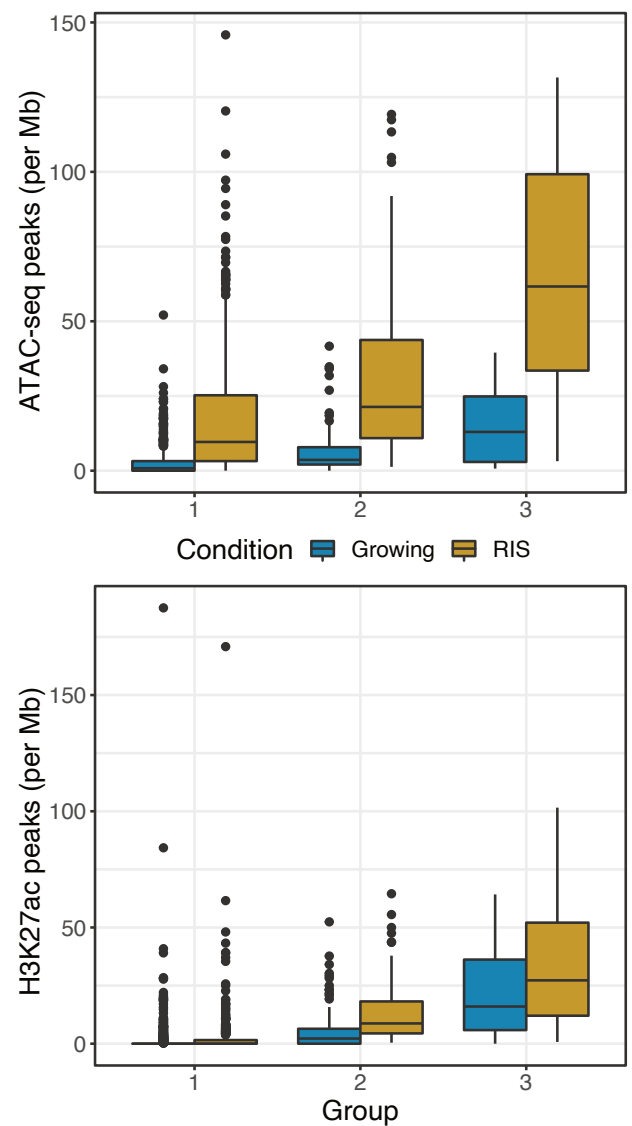

d

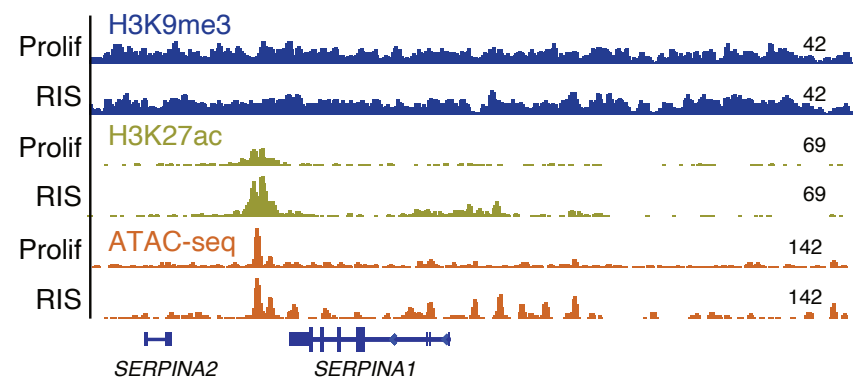

Extended Data Figure 3. H3K9me3 exhibits increases in accessibility and H3K27ac ChIP-seq signal in RIS. (a) ATAC-seq and H3K27ac THOR-normalized signal in proliferative and RIS cells centred on three classes of ATAC-seq peaks: proliferative-specific, RIS-specific, and common between the two conditions. (b) Number of ATAC-seq (top) and H3K27ac (bottom) peaks across the three groups of H3K9me3 peaks defined as permissive (group 2 and 3 ) and nonpermissive (group 1). The box plots correspond to the median, $25^{\text {th }}$ to $75^{\text {th }}$ percentiles, and the whiskers correspond to the $10^{\text {th }}$ to $90^{\text {th }}$ percentiles. (c) IGV tracks of genes of interest and position of SINE and Alu repeats (UCSC annotation) over their gene bodies. (d) Region surrounding the SERPINA1 gene characterised by H3K9me3 ChIP-seq signal, as well as protruding H3K27ac and ATAC-seq peaks. 


\section{Tomimatsu_Extended Data Fig. 4}
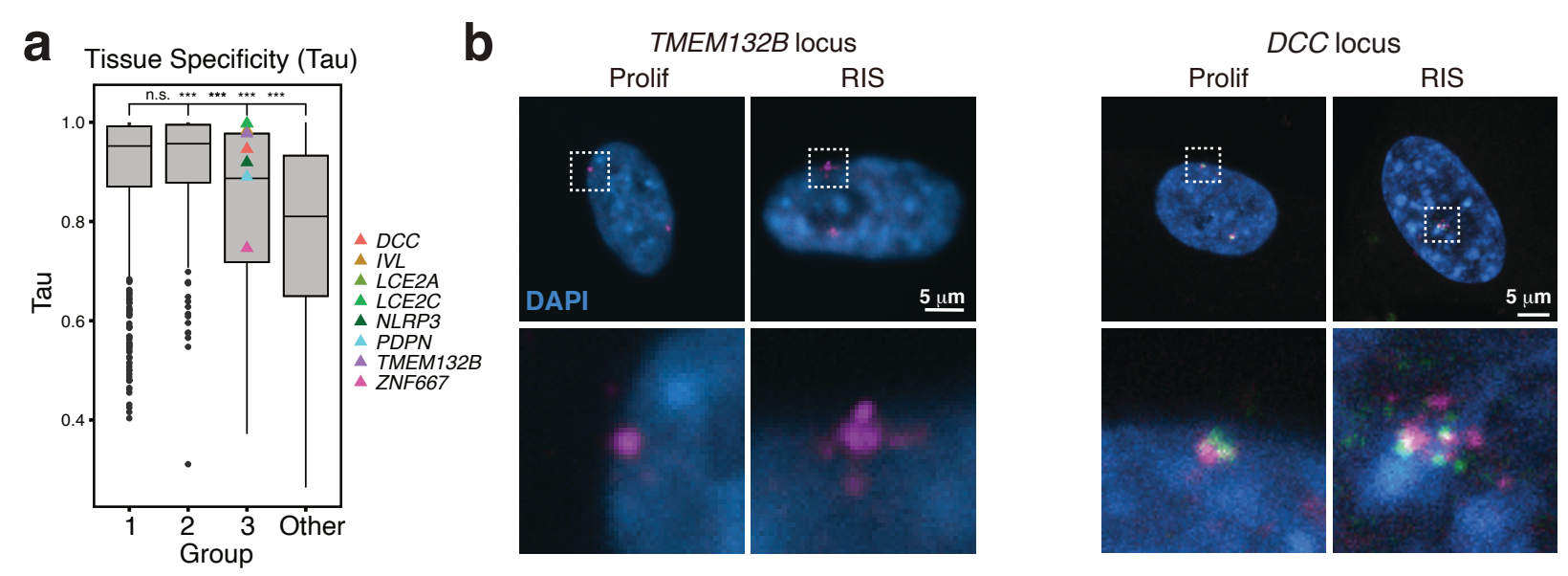

Extended Data Figure 4. Tissue specificity of genes in $\mathrm{H} 3 \mathrm{~K} 9 \mathrm{me} 3$ peaks. (a) Tissue specificity score of the genes within the three groups of H3K9me3 peaks against all other genes. The Tau score ranges from 0 - ubiquitous expression across tissues to 1 - tissue specific; the 8 genes of interest in group 3 are highlighted. Significance testing was performed with two-sided Student's ttests, ${ }^{* *} P<0.001$, n.s., not significant. The box plots correspond to the median, $25^{\text {th }}$ to $75^{\text {th }}$ percentiles, and the whiskers correspond to the $10^{\text {th }}$ to $90^{\text {th }}$ percentiles. (b) DCC (left) and TMEM132B (right) genes represented by multiprobe DNA-FISH signals in the proliferative and RIS conditions. Probe sets are shown in Fig. 5b. Note, single colour probe images are shown for TMEM132B. 


\section{Tomimatsu_Extended Data Fig. 5}

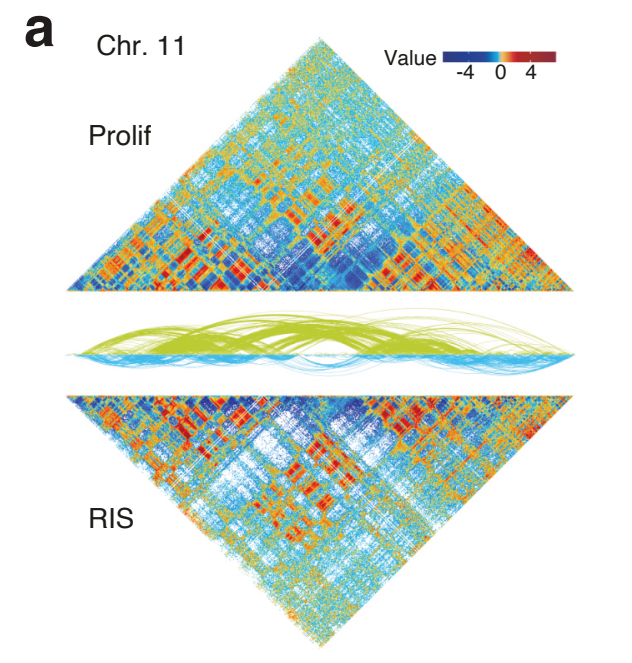

b

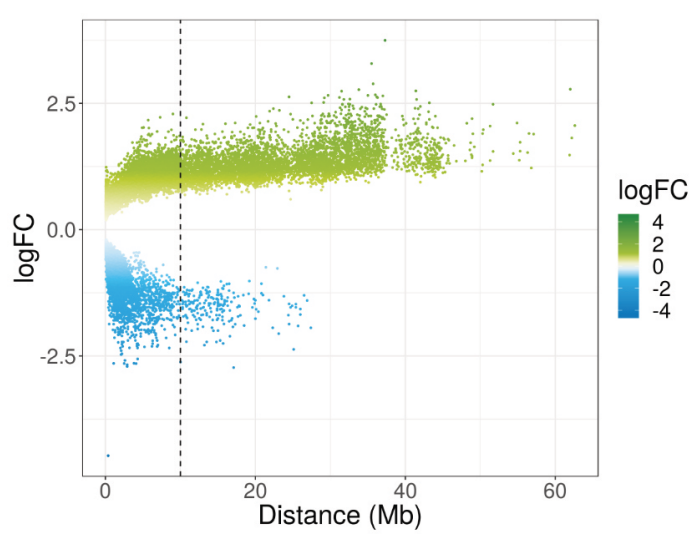

e

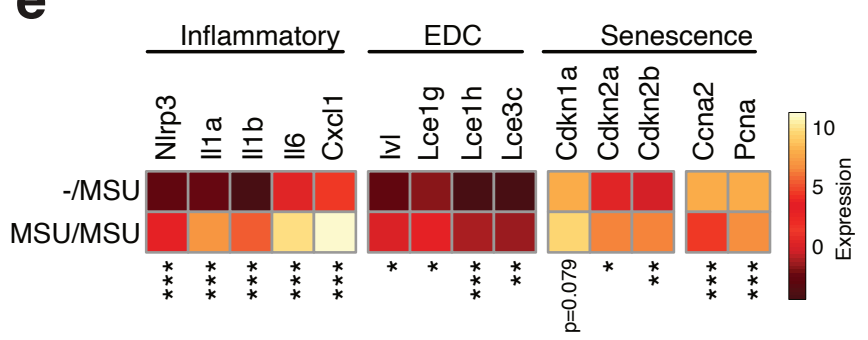

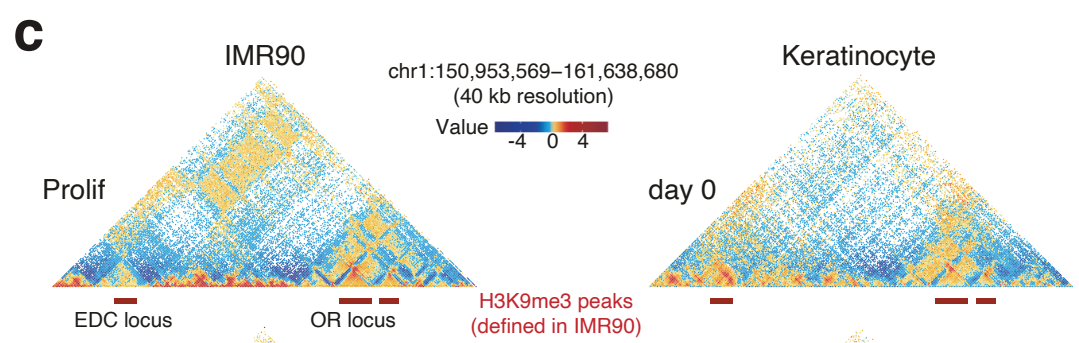

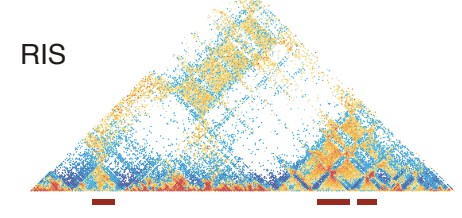

d
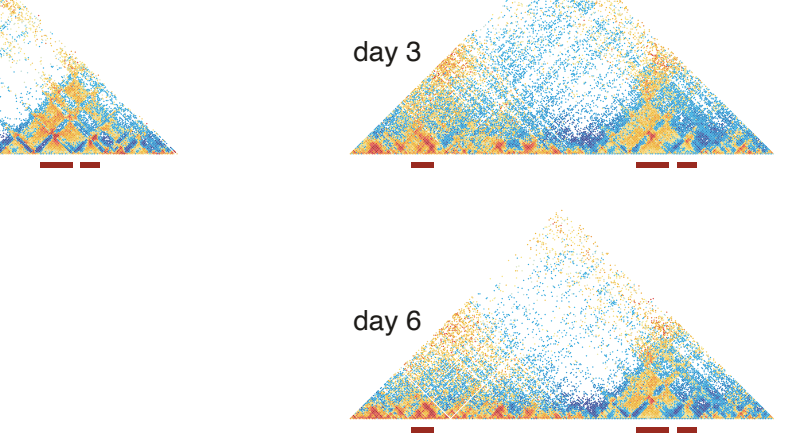

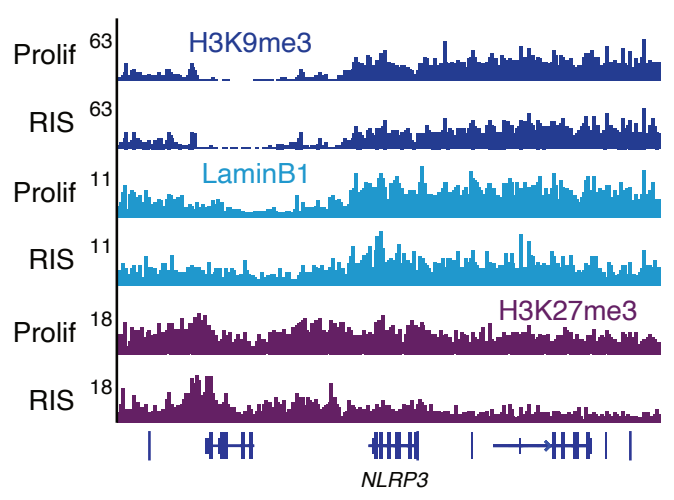

Extended Data Figure 5. Three-dimensional interaction changes occurring during RIS. (a) $200 \mathrm{~kb}$ resolution $\mathrm{Hi}-\mathrm{C}$ maps in proliferative and RIS cells as well as significant interaction changes between the two conditions, where green arcs represent increases and blue arcs represent decreases. (b) Significant H3K9me3-H3K9me3 interaction changes (200 kb bins): log-fold changes (logFC) against distance between the interacting regions, dotted line corresponds to $10 \mathrm{Mb}$ distance. (c) $\mathrm{Hi}-\mathrm{C}$ interaction profiles at $40 \mathrm{~kb}$ resolution in proliferating (Prolif) and RIS IMR90 cells as well as differentiating keratinocytes at the EDC/OR loci. (d) Epigenetic profile of the NLRP3 locus in terms of H3K9me3, LaminB1 and H3K27me3 THORnormalized ChIP-seq signal in proliferating and RIS cells. (e) Normalized gene expression (log-counts per million) of pro-inflammatory genes, EDC genes and senescence-representative genes in -/MSU and MSU/ MSU synovial fibroblasts. ${ }^{*}$ Adjusted $P<0.05,{ }^{* *} P<0.01,{ }^{* * *} P<0.001$. 